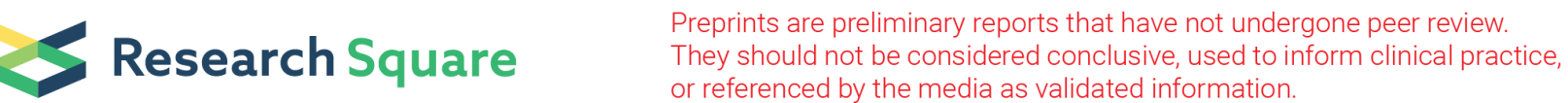

\section{Synthesis and Antimicrobial, Antiproliferative Evaluation of Novel Quinolone and Conazole Analogues via Conventional and Microwave Techniques}

\section{Şule Ceylan}

Artvin Coruh University: Artvin Coruh Universitesi

Yıldız Uygun Cebeci ( $\square$ yildizuygun41@hotmail.com )

Karadeniz Technical University: Karadeniz Teknik Universitesi

Neslihan Demirbaş

Karadeniz Technical University: Karadeniz Teknik Universitesi

Şengül Alpay Karaoğlu

Recep Tayyip Erdoğan University: Recep Tayyip Erdogan Universitesi

Muhammed Altun

Çankırı Karatekin Üniversitesi: Cankiri Karatekin Universitesi

\section{Research Article}

Keywords: 1,2,4-triazole, quinolone, mannich base, antimicrobial, anticancer activity

Posted Date: May 14th, 2021

DOl: https://doi.org/10.21203/rs.3.rs-505744/v1

License: (c) (1) This work is licensed under a Creative Commons Attribution 4.0 International License.

Read Full License 


\section{Abstract}

1,2,4-Triazole-3-one (3), acquired from cinnemaldehyde was converted to the corresponding carbox(thio)amides via several steps (6a-c). Their reaction with sodium hydroxide gave the 1,2,4-triazole derivatives (7a-c). Compound $\mathbf{3}$ treatment with 2-bromo-1-(4-chlorophenyl) ethanone or 2-chloro-1-(2,4dichlorophenyl)ethanone afforded the compounds $8 \mathrm{a}, \mathrm{b}$ and by reducing these compounds reduction products were obtained $(9 a, b)$. The synthesis of $(10 a-e)$ was carried out by the reaction compounds $9 a, b$ with different benzyl chlorides. Then oxadiazol derivative (12) was obtained by ring closure from hydrazide compound $\mathbf{5}$. Subsequently compounds $3,7 a-c$ and 12 were treated with various amines in the presence of formaldehyde to yield Mannich bases (11a-e, 14a-e, 13a,b). Microwave-assisted and conventional techniques were utilized for the syntheses. The structures of newly synthesized compounds were illuminated by spectroscopic methods. Their antimicrobial (MIC method), and anticancer activities (Abay's method) were examined. Results showed that most of the compounds exhibited good antimicrobial activities. Especially compounds $14 \mathrm{a}-\mathrm{e}$ which is a mannich base showed very good antitubercular activity against Mycobacterium smegmatis compared with Streptomycin standard drug. Also compounds $\mathbf{8 a}$ and $\mathbf{9 b}$ have been found to have strong antiproliferative effects on the HeLa cervical cancer cells and also these compounds did not have cytotoxic effect on normal cell.

\section{Introduction}

Cancer, is the result of uncontrolled growth of cells a major health concern and amongst the most important reasons of death worldwide [1-3]. The World Health Organization's cancer agency warns that there will be 22 million new cases of cancer every year within the next two decades [4]. Hence, cancer treatment is the primary task of today's medical research [5]. Despite the invention of several chemotherapeutic agents, still treatment of cancer is a major challenge, because of multi-drug resistance, toxicity or poor bioavailability. The effectiveness of many anticancer drugs is limited by acquired resistance to drugs, side effects due to their toxicity to normal cells because of their inability to differentiate between normal and cancerous cells. In this context, there is need to design and synthesize new, effective and less toxic anticancer agents throwing a challenge to medicinal chemists all over the World $[6,7]$.

Infectious diseases continue to be a leading threat to human health, and the rapid development of bacterial resistance to current antibiotic chemotherapies has rendered lots of therapy weapons less effective [8]. It is anticipated that antibiotic resistance is going to cause more than $10,000,000$ deaths per year by the year 2050, posing a formidable challenge for disease treatment as pathogens become resistant to clinical drugs [9]. The World Health Organization has launched a global action plan calling on all countries to take measures towards drug-resistant microbes, and the discovery of efficacious and safer antimicrobials with new or multiple mechanisms of action has been an urgent need to combat resistant strains $[10,11]$. 
Organic molecules bearing heterocyclic compounds have received a significant consideration in all fields of life comprising medicinal, pharmaceutical, combinatorial, natural resources, agriculture and dyes products $[3,12]$. Hybrid compounds are molecules with two or more structural fields, which have diverse dual activities and biological features. These substances generally possess the capacity to get over medicine resistance, develop pharmacokinetic structures, and abate toxicity $[13,14]$.

Triazoles are an important class of aromatic five-membered heterocyclic compounds with a wide variety of biological activities. They consist of two structural isomers: 1,2,3- and 1,2,4-triazole. Compounds based on 1,2,4-triazole scaffold show diverse biological and pharmacological activities [15-17]. The synthesized compounds having 1,2,4-triazole-3-thione skeleton have been reported to possess biological and pharmacological activities such as anticonvulsant, urease inhibition, antioxidant, analgesic. antiparasitic, antiulcer, anticancer, anti-HIV, anti-tuberculosis, antiamoebic, antigiardial, antiepileptic, antiinflammatory, antidepressant and anxiolytic [18-24].

Quinolines are widespread pharmacophores in the anti-bacterial substances. Fluoroquinolones are noted as one of the most broadly used antibacterial agents for the cure of bacterial illness. The cytotoxic activity of quinolone derivatives has become the source of new anticancer agents, which might also help addressing side-toxicity and resistance [25]. Quinolines and related derivatives are useful compounds with diverse pharmaceutical applications, and some have even reached markets for treatment of various ailments [26]. Thus, the hybridization of quinoline/quinolone with 1,2,4-triazole is inclined to present hopeful anti-bacterial agents $[27,28]$.

Imidazoles and triazoles, as a group called conazoles, are currently used worldwide as fungicides for grain, vegetable, fruit, and flower production and as pharmaceuticals for treatment of human mycoses including vaginal mycoses in pregnant women and thrush in infants [29].

Recently, multicomponent reactions (MCRs) have received considerable attention by synthetic organic and medicinal chemists for the construction of complex molecules having biological activity. When compared with conventional organic reactions, MCRs have some superior properties including high conversion rate, minimal reaction time and structural complexity. Thus, MCRs are also considered as green chemical processes [30]. Among these, Mannich reaction, a one pot three-component condensation reaction, provide synthetically and biologically important $\beta$-aminoalkylated compounds, which are important intermediates for the construction of various nitrogen-containing natural products and pharmaceuticals [31].

Microwave-assisted organic synthesis of various heterocyclic moieties is an effective and environmentfriendly synthetic approach and becoming an effective tool of green chemistry method. Microwave assisted techniques were reported to be more effective in perspective of environment, reaction time, high yields, ease of work-up and isolation of products. Moreover, solvents which are often expensive, toxic, difficult to remove in the case of aprotic dipolar solvents with high boiling point, and are environmentally polluting agents, are not necessary most of the microwave assisted synthesis [32, 33]. 
One of the main strategies for the discovery of new drugs is combining two or more pharmacophoric moieties in a single molecule to obtain the synergistic effect or to obtain antitumor agents that have a novel mode of action. For this purpose, various quinolone and conazole derivatives with biological activity were synthesized in this study.

\section{Material And Methods}

\subsection{Experimental}

\subsubsection{General}

The chemicals were obtained from Fluka Chemie AG Buchs (Switzerland) and utilized without further purification. Melting points of the synthesized molecules were found in open capillaries over a Büchi B540 melting degree device and are uncorrected. Reactions were screened byathin-layer chromatography (TLC) over silicagel 60 F254 aluminium plates. Mobile stage was ethyl acetate:diethyl ether (1:1), and finding was performed utilizing UV lamp. FT-IR datas were founded utilizing a Perkin Elmer 1600 serial FTIR spectrometer. ${ }^{13} \mathrm{C}$ NMR and ${ }^{1} \mathrm{H}$ NMR spectra were recorded in DMSO- $d_{6}$ on a BRUKER AVENE // 400 $\mathrm{MHz}$ NMR Spectrometer (400.13 MHz for ${ }^{1} \mathrm{H}$ and $100.62 \mathrm{MHz}$ for $\left.{ }^{13} \mathrm{C}\right)$. The chemical ranges are dedicated at ppm interested to $\mathrm{Me}_{4} \mathrm{Si}$ as an interior reference. $J$ valuesaare presented inaHz. Elemental assay was acquired over aaCostech ElementalaCombustion SystemaCHNS-O elementalaanalyzer. All the molecules present $\mathrm{C}, \mathrm{H}$ andaN assay within $\pm 0.4 \%$ a of theatheoretical ranges. The mass spectra were provided on a Quattro LC-MS(70 eV) Instrument.

\subsubsection{4-Amino-5-metil-2,4-dihidro-3H-1,2,4-triazol-3-on (2) [34]}

Hydrazine hydrate $(25 \mathrm{mmol})$ in $3 \%$ water solution was added to ethyl 2- (1-ethoxyethylidene) hydrazinecarboxylate (1) $(10 \mathrm{mmol})$ and boiled under reflux for 8 hours. After the mixture was left in the freezer overnight, the white solid which had precipitated was filtered off and purified by crystallization from ethanol.

Yield: 70\%, m.p: $210-212^{\circ} \mathrm{C}$. FT-IR $\left(\mathrm{U}_{\max }, \mathrm{cm}^{-1}\right)$ : 3295 ve $3207\left(\mathrm{NH}_{2}\right), 3218(\mathrm{NH}), 1683(\mathrm{C}=0), 1588(\mathrm{C}=$ N). ${ }^{1} \mathrm{H}$ NMR (DMSO-d $\left.{ }_{6}, \delta \mathrm{ppm}\right): 2.07\left(3 \mathrm{H}, \mathrm{s}, \mathrm{CH}_{3}\right), 5.13\left(2 \mathrm{H}, \mathrm{s}, \mathrm{NH}_{2}\right), 11.22(1 \mathrm{H}, \mathrm{s}, \mathrm{NH})$.

\subsubsection{5-methyl-4-\{[(1Z,2Z)-3-phenylprop-2-en-1- ylidene]amino-2,4-dihydro-3H-1,2,4-triazol-3-one (3) [34]}

In a round-bottom flask, a mixture of 1,2,4-triazol-3-one (2) (10 mmol) and cinnemaldehyde (10 mmol) was heated on oil bath at $110-120^{\circ} \mathrm{C}$ for 2 hours. The solid obtained by cooling the mixture to room temperature was crystallized from the ethanol: water (1: 3 ) mixture. The crystals obtained were purified 
by crystallizing several more times from the same solvent and after drying in vacuo, it was identified as compound 3.

Yield: 78\%, m.p: $190-192^{\circ} \mathrm{C}$. FT-IR $\left(\mathrm{U}_{\max } \mathrm{cm}^{-1}\right): 3167(\mathrm{NH}), 3041$ (aromatic $\left.\mathrm{CH}\right), 1687(\mathrm{C}=0), 1512(\mathrm{C}=$ N). ${ }^{1} \mathrm{H}$ NMR (DMSO- $d_{6}, \delta$ ppm): $2.20\left(3 \mathrm{H}, \mathrm{s}, \mathrm{CH}_{3}\right), 7.00(1 \mathrm{H}, \mathrm{t}, J=8.0 \mathrm{~Hz}, \mathrm{arH}), 7.04-7.40(3 \mathrm{H}, \mathrm{m}, \operatorname{arH}), 7.65$ $(1 \mathrm{H}, \mathrm{d}, J=8.0 \mathrm{~Hz}, \mathrm{arH}), 9.50(3 \mathrm{H}, \mathrm{s}, 3 \mathrm{CH}), 11.77(1 \mathrm{H}, \mathrm{s}, \mathrm{NH}) .{ }^{13} \mathrm{C}$ NMR (DMSO-d $\left.d_{6}, \delta \mathrm{ppm}\right): 11.65\left(\mathrm{CH}_{3}\right)$, $125.41(\mathrm{CH}), \operatorname{arC}:[128.02(2 \mathrm{CH}), 129.32(2 \mathrm{CH}), 129.98(\mathrm{CH}), 135.82(\mathrm{C})], 143.69(\mathrm{CH}), 144.54$ (triazole C3), 151.71 (triazole C-5), $156.64(\mathrm{CH})$. El MS m/z (\%): 135.28 (100), $113.14(80), 251.32\left([\mathrm{M}+\mathrm{Na}]^{+}, 49\right)$, 103.33 (37), 114.21 (36), 215.22 (28), 182.20 (25), 152.26 (21).

\subsubsection{Ethyl (3-methyl-5-oxo-4-\{[(1Z,2Z)-3-phenylprop-2-en- 1-ylidene]amino-4,5-dihydro-1 H-1,2,4-triazol-1-yl)acetate \\ (4)}

The solution of compound (3) $(10 \mathrm{mmol})$ in absolute ethanol was refluxed with sodium $(10 \mathrm{mmol})$ in absolute ethanol for $2 \mathrm{~h}$. Then, ethyl bromoacetate $(1.2 \mathrm{~mL}, 10 \mathrm{mmol})$ was added and refluxed for an additional $6 \mathrm{~h}$. After evaporation of solvent under reduced pressure, a solid appeared. The crude product was recrystallized from ethanol-water (1:1) to afford the desired compound.

Yield: 76\%, m.p: 85-87 C. FT IR $\left(\mathrm{U}_{\max } \mathrm{cm}^{-1}\right)$ : 3037 (aromatic $\left.\mathrm{CH}\right), 1734(\mathrm{C}=0), 1701(\mathrm{C}=0), 1170(\mathrm{C}-0)$, $1595(\mathrm{C}=\mathrm{N}) .{ }^{1} \mathrm{H}$ NMR (DMSO- $\left.d_{6}, \delta \mathrm{ppm}\right): 1.20-1.23\left(3 \mathrm{H}, \mathrm{m}, \mathrm{CH}_{3}\right), 2.26\left(3 \mathrm{H}, \mathrm{s}, \mathrm{CH}_{3}\right), 4.15-4.17(2 \mathrm{H}, \mathrm{m}$, $\left.\mathrm{CH}_{2}\right), 4.58\left(2 \mathrm{H}, \mathrm{s}, \mathrm{CH}_{2}\right), 7.04-7.10(1 \mathrm{H}, \mathrm{m}, \mathrm{arH}), 7.34-7.42(4 \mathrm{H}, \mathrm{m}, \mathrm{arH}), 7.68-7.70(2 \mathrm{H}, \mathrm{m}, 2 \mathrm{CH}), 9.46(1 \mathrm{H}$, $\mathrm{d}, J=8.0 \mathrm{~Hz}, \mathrm{CH}) .{ }^{13} \mathrm{C} \mathrm{NMR}\left(\mathrm{DMSO}_{-}, \delta \mathrm{ppm}\right): 11.51\left(\mathrm{CH}_{3}\right), 14.46\left(\mathrm{CH}_{3}\right), 46.77\left(\mathrm{CH}_{2}\right), 61.69\left(\mathrm{CH}_{2}\right), 125.08$ $(\mathrm{CH}), 125.10(\mathrm{CH}), 128.48(\mathrm{CH})$, arC: [128.16 (CH), $129.35(\mathrm{CH}), 129.37(\mathrm{CH}), 130.14(\mathrm{CH}), 130.17(\mathrm{CH})$, 144.00 (C)], 150.20 (triazole C-3), 157.38 (triazole C-5), 168.13 (C= 0). El MS $m / z(\%): 337.48\left([\mathrm{M}+\mathrm{Na}]^{+}\right.$, 100), $338.43\left([\mathrm{M}+\mathrm{Na}]^{+}\right.$, 38). Elemental analysis for: $\mathrm{C}_{16} \mathrm{H}_{18} \mathrm{~N}_{4} \mathrm{O}_{3}$; Calculated (\%): $\mathrm{C}, 61.13 ; \mathrm{H}, 5.77 ; \mathrm{N}$, 17.82; Found (\%): C, 61.45; H, 5.80; N, 17.90.

\subsubsection{2-(3-methyl-5-oxo-4-\{[(1Z,2Z)-3-phenylprop-2-en-1- ylidene]amino\}-4,5-dihydro-1 $\mathrm{H}-1,2,4$-triazol-1- yl)acetohydrazide (5)}

Aamixture of molecule 4 (10 mmol) inaethanol wasarefluxed withahydrazine hydrate $(25 \mathrm{mmol})$ fora $15 \mathrm{~h}$. The solid acquired on keeping the reaction solution inacold wasafiltered off, recrystallizedafrom ethylaacetate:diethyl ethera(1:3) toaafford the desiredaproduct $\mathbf{5}$.

Yield: $78 \%$, m.p: $177-179^{\circ} \mathrm{C}$. FT IR $\left(\mathrm{U}_{\max } \mathrm{cm}^{-1}\right)$ : $3337(\mathrm{NH}), 3280\left(\mathrm{NH}_{2}\right), 3068$ (aromatic $\left.\mathrm{CH}\right), 1698(\mathrm{C}=$ 0), 1655 (C= ) ), $1578(\mathrm{C}=\mathrm{N}) .{ }^{1} \mathrm{H}$ NMR (DMSO-d 6 , $\left.\delta \mathrm{ppm}\right): 2.08\left(3 \mathrm{H}, \mathrm{d}, J=8.0 \mathrm{~Hz}, \mathrm{CH}_{3}\right), 4.18\left(2 \mathrm{H}, \mathrm{s}, \mathrm{CH}_{2}\right)$, $5.25\left(2 \mathrm{H}, \mathrm{s}, \mathrm{NH}_{2}\right), 6.08-7.03(5 \mathrm{H}, \mathrm{m}, \mathrm{arH}), 8.55(3 \mathrm{H}, \mathrm{s}, 3 \mathrm{CH}), 9.17(1 \mathrm{H}, \mathrm{s}, \mathrm{NH}) .{ }^{13} \mathrm{C}$ NMR (DMSO- $\left.d_{6}, \delta \mathrm{ppm}\right)$ : $11.07\left(\mathrm{CH}_{3}\right), 46.79\left(\mathrm{CH}_{2}\right), 77.90(2 \mathrm{CH}), 84.80(\mathrm{CH}), \operatorname{arC}:[100.80(\mathrm{CH}), 101.20(2 \mathrm{CH}), 122.78(2 \mathrm{CH}), 145.46$ 
(C)], 153.69 (triazole C-3), 166.49 (triazole C-5), $170.68(\mathrm{C}=0)$. El MS $m / z(\%): 323.21\left([\mathrm{M}+\mathrm{Na}]^{+}, 100\right)$, 355.22 (18), 363.27 (16). Elemental analysis for: $\mathrm{C}_{14} \mathrm{H}_{16} \mathrm{~N}_{6} \mathrm{O}_{2}$; Calculated (\%): C, 55.99; $\mathrm{H}, 5.37 ; \mathrm{N}, 27.98$; Found (\%): C, 56.01; H, 5.40; N, 28.00.

General Method for the Synthesis of Compounds 6a-c

Phenyl isothiocyanate (for $\mathbf{6 a}$ ), benzyl isothiocyanate (for $\mathbf{6 b}$ ) or phenyl isocyanate (for $\mathbf{6 c}$ ) (20 mmol)awas addedato the mixture of molecule $5(10 \mathrm{mmol})$ inadried dichloro methaneadrop wise and the solution was stirred ataroom temperatureafor $24 \mathrm{~h}$. The solid product was precipitated.

\subsubsection{2-[(3-methyl-5-oxo-4-\{[(1E,2Z)-3-phenylprop-2-en-1- ylidene]amino-4,5-dihydro-1H-1,2,4-triazol-1-yl)acetyl]-N- phenylhydrazinecarbothioamide (6a)}

Yield: $91 \%$, m.p. $148-149^{\circ} \mathrm{C}$. FT IR $\left(\mathrm{U}_{\max }, \mathrm{cm}^{-1}\right): 3349(\mathrm{NH}), 3283(2 \mathrm{NH}), 3061$ (aromatic $\left.\mathrm{CH}\right), 2924$ (alifatic CH), $1715(\mathrm{C}=0), 1543(\mathrm{C}=\mathrm{N}), 1240(\mathrm{C}=\mathrm{S}) .{ }^{1} \mathrm{H}$ NMR (DMSO- $\left.d_{6}, \delta \mathrm{ppm}\right): 1.84\left(3 \mathrm{H}, \mathrm{s}, \mathrm{CH}_{3}\right), 4.48$ $\left(2 \mathrm{H}, \mathrm{d}, J=4.0 \mathrm{~Hz}, \mathrm{CH}_{2}\right), 4.67\left(2 \mathrm{H}, \mathrm{s}, \mathrm{CH}_{2}\right), 7.02-7.15(8 \mathrm{H}, \mathrm{m}, \mathrm{arH}), 7.42(2 \mathrm{H}, \mathrm{d}, J=8.0 \mathrm{~Hz}, \mathrm{arH}), 8.28(1 \mathrm{H}, \mathrm{s}$, $\mathrm{CH}), 9.16(1 \mathrm{H}, \mathrm{s}, \mathrm{CH}), 9.25(1 \mathrm{H}, \mathrm{s}, \mathrm{CH}), 9.91(1 \mathrm{H}, \mathrm{s}, \mathrm{NH}), 11.28(1 \mathrm{H}, \mathrm{s}, \mathrm{NH}), 11.38(1 \mathrm{H}, \mathrm{s}, \mathrm{NH}) .{ }^{13} \mathrm{C} \mathrm{NMR}$ (DMSO-d 6 , $\delta$ ppm): $11.53\left(\mathrm{CH}_{3}\right), 46.76\left(\mathrm{CH}_{2}\right), 90.76(\mathrm{CH}), 98.86(\mathrm{CH}), 100.73(\mathrm{CH})$, $\operatorname{arC}:[125.15(\mathrm{CH})$, $127.13(\mathrm{CH}), 127.40(\mathrm{CH}), 128.15(2 \mathrm{CH}), 129.39(\mathrm{CH}), 130.16(2 \mathrm{CH}), 135.75(2 \mathrm{CH}), 140.84(\mathrm{C}), 143.81(\mathrm{C})]$, 150.40 (triazole $\mathrm{C}-3), 157.14$ (triazole $\mathrm{C}-5), 178.20(\mathrm{C}=0), 189.54(\mathrm{C}=\mathrm{S})$. El MS $\mathrm{m} / z(\%): 473.23(40)$, $472.17\left([\mathrm{M}+\mathrm{Na}]^{+}, 100\right), 437.25$ (35). Elemental analysis for: $\mathrm{C}_{22} \mathrm{H}_{23} \mathrm{~N}_{7} \mathrm{O}_{2} \mathrm{~S}$; Calculated (\%): C, 58.78; $\mathrm{H}$, $5.16 ; \mathrm{N}, 21.81$; Found (\%): C, 58.80; H, 5.20; N, 21.88.

\subsubsection{N-benzyl-2-[(3-methyl-5-oxo-4-\{[(1E,2Z)-3- phenylprop-2-en-1-ylidene]amino-4,5-dihydro-1 $1+1,2,4-$ triazol-1-yl)acetyl]hydrazinecarbothioamide (6b)}

Yield: $91 \%$, m.p. $148-149^{\circ} \mathrm{C}$. FT IR $\left(\mathrm{U}_{\max } \mathrm{cm}^{-1}\right): 3349(\mathrm{NH}), 3283(2 \mathrm{NH}), 2924$ (alifatic $\left.\mathrm{CH}\right), 3061$ (aromatic CH), $1715(\mathrm{C}=0), 1543(\mathrm{C}=\mathrm{N}), 1240(\mathrm{C}=\mathrm{S}) .{ }^{1} \mathrm{H}$ NMR $\left(\mathrm{DMSO}-d_{6}, \delta \mathrm{ppm}\right): 1.84\left(3 \mathrm{H}, \mathrm{s}, \mathrm{CH}_{3}\right), 4.48$ $\left(2 \mathrm{H}, \mathrm{d}, J=4.0 \mathrm{~Hz}, \mathrm{CH}_{2}\right), 4.67\left(2 \mathrm{H}, \mathrm{s}, \mathrm{CH}_{2}\right), 7.02-7.15(8 \mathrm{H}, \mathrm{m}, \mathrm{arH}), 7.42(2 \mathrm{H}, \mathrm{d}, J=8.0 \mathrm{~Hz}, \operatorname{arH}), 8.28(1 \mathrm{H}, \mathrm{s}$, $\mathrm{CH}), 9.16(1 \mathrm{H}, \mathrm{s}, \mathrm{CH}), 9.25(1 \mathrm{H}, \mathrm{s}, \mathrm{CH}), 9.91(1 \mathrm{H}, \mathrm{s}, \mathrm{NH}), 11.28(1 \mathrm{H}, \mathrm{s}, \mathrm{NH}), 11.38(1 \mathrm{H}, \mathrm{s}, \mathrm{NH}) .{ }^{13} \mathrm{C} \mathrm{NMR}$ (DMSO-d 6 , $\delta$ ppm): $11.53\left(\mathrm{CH}_{3}\right), 46.76\left(\mathrm{CH}_{2}\right), 47.11\left(\mathrm{CH}_{2}\right), 90.76(\mathrm{CH}), 98.86(\mathrm{CH}), 100.73(\mathrm{CH})$, arC: [125.15 (CH), $127.13(\mathrm{CH}), 127.40(\mathrm{CH}), 128.15(\mathrm{CH}), 129.39(\mathrm{CH}), 130.16(2 \mathrm{CH}), 135.75(2 \mathrm{CH}), 140.84$ (C), 143.81 (C)], 150.40 (triazole C-3), 157.14 (triazole C-5), $178.20(\mathrm{C}=0), 189.54(\mathrm{C}=\mathrm{S})$. El MS $\mathrm{m} / \mathrm{z}(\%)$ : $472.17\left([\mathrm{M}+\mathrm{Na}]^{+}, 100\right), 473.23(40), 437.25$ (35). Elemental analysis for: $\mathrm{C}_{22} \mathrm{H}_{23} \mathrm{~N}_{7} \mathrm{O}_{2} \mathrm{~S}$; Calculated (\%): $\mathrm{C}$, 58.78; H, 5.16; N, 21.81; Found (\%): C, 58.80; H, 5.20; N, 21.88.

\subsubsection{2-[(3-methyl-5-oxo-4-\{[(1E,2Z)-3-phenylprop-2-en-1- ylidene]aminot-4,5-dihydro-1 H-1,2,4-triazol-1-yl)acetyl]-N-}




\section{phenylhydrazinecarboxamide (6c)}

Yield: 93\%, m.p. $152-154^{\circ} \mathrm{C}$. FT IR (umax, cm-1): $3360(\mathrm{NH}), 3198(2 \mathrm{NH}), 3037$ (aromatic CH), $1715(\mathrm{C}=$ 0), $1626(\mathrm{C}=\mathrm{S}), 1577(\mathrm{C}=\mathrm{N}) .{ }^{1} \mathrm{H}$ NMR (DMSO-d $\left.6, \delta \mathrm{ppm}\right): 2.25\left(3 \mathrm{H}, \mathrm{s}, \mathrm{CH}_{3}\right), 4.46\left(2 \mathrm{H}, \mathrm{s}, \mathrm{CH}_{2}\right), 6.97-7.28$ (4H, m, arH), 7.33-7.47 (4H, m, arH), $7.69(2 \mathrm{H}, \mathrm{d}, J=4.0 \mathrm{~Hz}, \mathrm{arH}), 8.26(2 \mathrm{H}, \mathrm{s}, 2 \mathrm{CH}), 8.78(1 \mathrm{H}, \mathrm{s}, \mathrm{CH}), 9.50$ $(2 \mathrm{H}, \mathrm{s}, \mathrm{NH}), 10.04(1 \mathrm{H}, \mathrm{s}, \mathrm{NH}) \cdot{ }_{13} \mathrm{C} \mathrm{NMR}\left(\mathrm{DMSO}-\mathrm{d}_{6}, \delta \mathrm{ppm}\right): 11.56\left(\mathrm{CH}_{3}\right), 46.78\left(\mathrm{CH}_{2}\right), 91.75(\mathrm{CH}), 97.56$ $(\mathrm{CH}), 101.43(\mathrm{CH})$, arC: [118.93 (CH), $119.11(\mathrm{CH}), 122.51(\mathrm{CH}), 122.73(\mathrm{CH}), 125.18(\mathrm{CH}), 129.10(\mathrm{CH})$, $129.16(\mathrm{CH}), 129.24(\mathrm{CH}), 129.38(\mathrm{CH}), 139.95(\mathrm{C}), 143.85(\mathrm{C})], 155.54$ (triazole C-3), 157.09 (triazole C-5), 166.90 (C= 0), 173.56 (C= 0). El MS m/z (\%): 442.31 ([M + Na]+, 100), 134.98 (56), 447.25 (31), 301.28 (25), $420.35([\mathrm{M}+1]+, 19)$. Elemental analysis for: $\mathrm{C}_{22} \mathrm{H}_{25} \mathrm{~N}_{7} \mathrm{O}_{3}$; Calculated (\%):C, 60.13; $\mathrm{H}, 5.05 ; \mathrm{N}, 23.38$; Found (\%): C, 60.17; H, 5.01; N, 23.40 .

General Method for the Synthesis of Compounds 7a-c

Method 1. A solution of the corresponding compound $6 a-c(10 \mathrm{mmol})$ in ethanol and water (1:1) was refluxed in the presence $\mathrm{NaOH}(2 \mathrm{~N})$ for $6 \mathrm{~h}$. Then the resulting solution was cooled to room temperature and acidified to $\mathrm{pH} 4$ with $37 \% \mathrm{HCl}$. The precipitate formed was filtered off, washed with water, and recrystallized from ethyl acetate to afford the desired compound.

Method 2. The mixture of the corresponding compound $6 \mathrm{a}-\mathrm{c}(1 \mathrm{mmol})$ and $\mathrm{NaOH}(0.5 \mathrm{mmol})$ in ethanol $(10 \mathrm{~mL})$ was irradiated in monomode microwave reactor in closed vessel with the pressure control at $100^{\circ} \mathrm{C}$ for $16 \mathrm{~min}$ (hold time) at $150 \mathrm{~W}$ maximum power (the progress of the reaction was monitored by TLC). Then the resulting solution was cooled to room temperature and acidified to $\mathrm{pH} 5$ with $37 \% \mathrm{HCl}$. The precipitate formed was filtered off, wash with water, and recrystallized from ethyl acetate (for 7a), ethanol (for $7 \mathrm{~b}$ and $7 \mathrm{c}$ ) to give the target compounds.

\subsubsection{2-[(5-mercapto-4-phenyl-4H-1,2,4-triazol-3- yl)methyl]-5-methyl-4-\{[(1E,2Z)-3-phenyl prop-2-en-1- ylidene]amino\}-2,4-dihydro-3H-1,2,4-triazol-3-one (7a)}

Yield: 65\% (Method 1), 80\% (Method 2), m.p. 203-204 ${ }^{\circ} \mathrm{C}$. FT IR $\left(\mathrm{U}_{\max }, \mathrm{cm}^{-1}\right): 3029(\operatorname{ar}-\mathrm{CH}), 2932(\mathrm{SH})$, 1680 (C = 0), 1585 (C = N). ${ }^{1} \mathrm{H}$ NMR (DMSO- $\left.d_{6}, \delta \mathrm{ppm}\right): 2.02\left(3 \mathrm{H}, \mathrm{s}, \mathrm{CH}_{3}\right), 5.08\left(2 \mathrm{H}, \mathrm{d}, J=8.0 \mathrm{~Hz}, \mathrm{CH}_{2}\right), 6.85$ $(2 \mathrm{H}, \mathrm{s}, \mathrm{arH}), 7.01-7.95(8 \mathrm{H}, \mathrm{m}, \mathrm{arH}), 8.76(1 \mathrm{H}, \mathrm{s}, \mathrm{CH}), 9.52(2 \mathrm{H}, \mathrm{s}, 2 \mathrm{CH}), 13.70(1 \mathrm{H}, \mathrm{s}, \mathrm{SH}) .{ }^{13} \mathrm{C}$ NMR (DMSO$\left.d_{6}, \delta \mathrm{ppm}\right): 10.11\left(\mathrm{CH}_{3}\right), 48.67\left(\mathrm{CH}_{2}\right), 91.80(\mathrm{CH}), 95.98(\mathrm{CH}), 100.56(\mathrm{CH}), \operatorname{arC}:[121.04(2 \mathrm{CH}), 125.36$ $(\mathrm{CH}), 126.10(\mathrm{CH}), 127.65(\mathrm{CH}), 128.93(\mathrm{CH}), 129.25(\mathrm{CH}), 130.67(\mathrm{CH}), 13212(2 \mathrm{CH}), 133.07(\mathrm{C}), 135.58$ (C)], 152.64 (triazole C-3), 156.19 (triazole C-3), 166.40 (triazole C-5), 175.25 (triazole C-5). El MS $\mathrm{m} / \mathrm{z}(\%)$ : $454.26\left([\mathrm{M}+\mathrm{Na}]^{+}, 31\right), 428.23$ (100), 406.27 (37), 287.27 (44), 261.24 (87), 187.16 (81). Elemental analysis for: $\mathrm{C}_{22} \mathrm{H}_{21} \mathrm{~N}_{7} \mathrm{OS}$; Calculated (\%): $\mathrm{C}, 61.23 ; \mathrm{H}, 4.91 ; \mathrm{N}, 22.72 ;$ Found (\%): $\mathrm{C}, 61.27 ; \mathrm{H}, 4.95 ; \mathrm{N}$, 22.75 . 


\subsubsection{2-[(4-benzyl-5-mercapto-4H-1,2,4-triazol-3- yl)methyl]-5-methyl-4-\{[(1E,2Z)-3-phenyl prop-2-en-1- ylidene]amino-2,4-dihydro-3H-1,2,4-triazol-3-one (7b)}

Yield: 68\% (Method 1), 86\% (Method 2), m.p. $198-199^{\circ} \mathrm{C}$. FT IR ( $\mathrm{U}_{\max } \mathrm{cm}^{-1}$ ): 3032 (aromatic $\left.\mathrm{CH}\right), 2929$ $(\mathrm{SH}), 1699$ (C = 0), $1598(\mathrm{C}=\mathrm{N}) .{ }^{1} \mathrm{H}$ NMR (DMSO- $\left.d_{6}, \delta \mathrm{ppm}\right): 2.02\left(3 \mathrm{H}, \mathrm{s}, \mathrm{CH}_{3}\right), 5.00(2 \mathrm{H}, \mathrm{d}, J=8.0 \mathrm{~Hz}$, $\left.\mathrm{CH}_{2}\right), 5.21\left(2 \mathrm{H}, \mathrm{d}, J=12.0 \mathrm{~Hz}, \mathrm{CH}_{2}\right), 6.92(2 \mathrm{H}, \mathrm{d}, J=8.0 \mathrm{~Hz}, \operatorname{arH}), 7.10-7.80(8 \mathrm{H}, \mathrm{m}, \operatorname{arH}), 8.73(1 \mathrm{H}, \mathrm{s}, \mathrm{CH})$, $9.34(2 \mathrm{H}, \mathrm{s}, 2 \mathrm{CH}), 14.02(1 \mathrm{H}, \mathrm{s}, \mathrm{SH}) .{ }^{13} \mathrm{C}$ NMR (DMSO-d $\left.6, \delta \mathrm{ppm}\right): 11.12\left(\mathrm{CH}_{3}\right), 46.38\left(\mathrm{CH}_{2}\right), 50.20\left(\mathrm{CH}_{2}\right)$, $93.70(\mathrm{CH}), 98.58(\mathrm{CH}), 103.48(\mathrm{CH}), \operatorname{arC}:[126.04(2 \mathrm{CH}), 127.45(\mathrm{CH}), 128.20(\mathrm{CH}), 128.40(\mathrm{CH}), 128.83$ $(\mathrm{CH}), 129.38(\mathrm{CH}), 129.52(\mathrm{CH}), 132.09(2 \mathrm{CH}), 133.64(\mathrm{C}), 134.78(\mathrm{C})], 155.54$ (triazole C-3), 157.09 (triazole C-3), 167.20 (triazole C-5), 172.20 (triazole C-5). El MS m/z (\%): 428.23 (100), 261.24 (87), 187.16 (81), 287.27 (44), 406.27 (37), $454.26\left([\mathrm{M}+\mathrm{Na}]^{+}, 31\right)$. Elemental analysis for: $\mathrm{C}_{23} \mathrm{H}_{25} \mathrm{~N}_{7} \mathrm{OS}$; Calculated (\%): C, 61.23; H, 4.91; N, 22.72; Found (\%): C, 61.27; H, 4.95; N, 22.75.

\subsubsection{5-methyl-2-[(5-oxo-4-phenyl-4,5-dihydro-1H-1,2,4- triazol-3-yl)methyl]-4-\{[(1E,2Z)-3-phenylprop-2-en-1- ylidene]amino\}-2,4-dihydro-3H-1,2,4-triazol-3-one (7c)}

Yield: $57 \%$ (Method 1), 76\% (Method 2), m.p. 188-190 C. FT IR $\left(\mathrm{U}_{\max } \mathrm{cm}^{-1}\right): 3370(\mathrm{NH}), 3027$ (aromatic $\mathrm{CH}), 1683(\mathrm{C}=0), 1626(\mathrm{C}=0), 1594(\mathrm{C}=\mathrm{N}) .{ }^{1} \mathrm{H}$ NMR (DMSO-d,$\left.\delta \mathrm{ppm}\right): 2.20\left(3 \mathrm{H}, \mathrm{s}, \mathrm{CH}_{3}\right), 4.53(2 \mathrm{H}, \mathrm{s}$, $\left.\mathrm{CH}_{2}\right), 7.29(2 \mathrm{H}, \mathrm{d}, \mathrm{J}=8.0 \mathrm{~Hz}, \mathrm{arH}), 7.31-7.65(8 \mathrm{H}, \mathrm{m}, \mathrm{arH}), 7.67(1 \mathrm{H}, \mathrm{s}, \mathrm{CH}), 7.85(1 \mathrm{H}, \mathrm{s}, \mathrm{CH}), 7.97(1 \mathrm{H}, \mathrm{s}$, $\mathrm{CH}), 10.77(1 \mathrm{H}, \mathrm{s}, \mathrm{NH}) .{ }^{13} \mathrm{C}$ NMR (DMSO- $\left.d_{6}, \delta \mathrm{ppm}\right): 11.40\left(\mathrm{CH}_{3}\right), 47.25\left(\mathrm{CH}_{2}\right), 97.70(\mathrm{CH}), 99.74(\mathrm{CH})$, 102.47 (CH), arC: [118.48 (CH), $119.70(\mathrm{CH}), 120.33(\mathrm{CH}), 122.93(\mathrm{CH}), 127.22(\mathrm{CH}), 127.47(\mathrm{CH}), 128.83$ $(\mathrm{CH}), 128.90(\mathrm{CH}), 129.00(\mathrm{CH}), 134.85(\mathrm{C}), 139.53(\mathrm{C})], 154.53$ (triazole C-3), 158.10 (triazole C-3), 168.32 (triazole C-5), 169.78 (triazole C-5). El MS $m / z(\%): 402.26$ ([M+1] $\left.]^{+}, 100\right), 288.21$ (95), 376.24 (87), 416.28 (76). Elemental analysis for: $\mathrm{C}_{22} \mathrm{H}_{23} \mathrm{~N}_{7} \mathrm{O}_{2}$; Calculated (\%): C, 62.83; $\mathrm{H}, 4.77 ; \mathrm{N}, 24.42 ;$ Found (\%): $\mathrm{C}, 62.85$; H, 4.79; N, 24.50.

General Method for The Synthesis of Compounds 8a,b

Method 1. The solution of compound $3(10 \mathrm{mmol})$ in ethanol was refluxed in the presence of sodium ethoxide (10 mmol) for $6 \mathrm{~h}$. Then, 2-bromo-1-(4-chlorophenyl)ethanone (10 mmol), (for 8a), 2-chloro-1(2,4-dichlorophenyl)ethanone $(10 \mathrm{mmol})$ (for $8 \mathrm{~b})$, was added into it, and the mixture was refluxed for additional $18 \mathrm{~h}$. After evaporation the solvent under reduced pressure, a solid appeared. This crude product was recrystallized from acetone:water (1:3) to give the target compound.

Method 2. The mixture of compound $3(1 \mathrm{mmol})$ and sodium ethoxyde $(1 \mathrm{mmol})$ in $10 \mathrm{~mL}$ of ethanol was irradiated in monomode microwave reactor in closed vessel with the pressure control at $120^{\circ} \mathrm{C}$ for $6 \mathrm{~min}$ (hold time) at $150 \mathrm{~W}$ maximum power. Then, 2 Then, 2-bromo-1-(4-chlorophenyl)ethanone (10 mmol), (for 
8a), 2-chloro-1-(2,4-dichlorophenyl)ethanone (10 mmol) (for $8 \mathrm{~b})$, was added into it, and the mixture was irradiated for additional 10 min under the same conditions. After removing the solvent under reduced pressure, a solid appeared.

\subsubsection{2-[2-(4-chlorophenyl)-2-oxoethyl]-5-methyl-4-\{(1Z,2Z)-3-phenylprop-2-en-1-ylidene] amino\}-2,4- dihydro-3H-1,2,4-triazol-3-one (8a)}

Yield: 65\% (Method 1), 82\% (Method 2), m.p: $125-127^{\circ} \mathrm{C}$. FT-IR $\left(\mathrm{U}_{\max }, \mathrm{cm}^{-1}\right)$ ) 3058 (aromatic CH), 1693 $(\mathrm{C}=0), 1625(\mathrm{C}=0) .{ }^{1} \mathrm{H}$ NMR (DMSO- $\left.d_{6}, \delta \mathrm{ppm}\right): 1.03-1.16\left(3 \mathrm{H}, \mathrm{m}, \mathrm{CH}_{3}\right), 5.38\left(2 \mathrm{H}, \mathrm{s}, \mathrm{CH}_{2}\right), 7.27-7.38$ $(1 \mathrm{H}, \mathrm{m}, \mathrm{arH}), 7.38-7.40(5 \mathrm{H}, \mathrm{m}, \mathrm{arH}), 7.64-7.67(3 \mathrm{H}, \mathrm{m}, \mathrm{arH}), 9.47-9.53(3 \mathrm{H}, \mathrm{m}, 3 \mathrm{CH}) .{ }^{13} \mathrm{C}$ NMR (DMSO-d $d_{6}$ $\delta$ ppm): $11.55\left(\mathrm{CH}_{3}\right), 52.14\left(\mathrm{CH}_{2}\right), 125.15(\mathrm{CH}), \operatorname{arC}:[125.45(\mathrm{CH}), 128.70(\mathrm{CH}), 128.95(\mathrm{CH}), 129.15(\mathrm{CH})$, $129.25(\mathrm{CH}), 129.34(\mathrm{CH}), 129.36(\mathrm{CH}), 129.53(\mathrm{CH}), 129.99(\mathrm{CH}), 139.52(\mathrm{C}), 144.30(\mathrm{C}), 144.48(\mathrm{C})]$, $144.02(\mathrm{CH}), 144.37(\mathrm{CH}), 150.63$ (triazole $\mathrm{C}-3), 151.87$ (triazole $\mathrm{C}-5), 192.56(\mathrm{C}=0)$. El MS $\mathrm{m} / \mathrm{z}(\%)$ : $403.39\left([\mathrm{M}+\mathrm{Na}]^{+}, 100\right), 405.40(37), 251.35(34), 449.38(33), 341.39(18), 381.37\left([\mathrm{M}+1]^{+}, 12\right)$.

2.1.1.12 2-[2-(2,4-dichlorophenyl)-2-oxoethyl]-5-methyl-4-\{(1Z,2Z)-3-phenylprop-2-en-1-ylidene ]amino\}-2,4dihydro-3H-1,2,4-triazol-3-one (8b)

Yield: 58\% (Method 1), 77\% (Method 2), m.p: 110-112 C. FT-IR ( $\left.\mathrm{U}_{\max }, \mathrm{cm}^{-1}\right): 3041$ (aromatic CH), 1689 $(\mathrm{C}=0), 1625(\mathrm{C}=0) .{ }^{1} \mathrm{H}$ NMR (DMSO-d $\left.d_{6}, \delta \mathrm{ppm}\right): 2.21\left(3 \mathrm{H}, \mathrm{s}, \mathrm{CH}_{3}\right), 5.25\left(2 \mathrm{H}, \mathrm{s}, \mathrm{CH}_{2}\right), 7.29-7.33(1 \mathrm{H}, \mathrm{m}$, arH), 7.38-7.42 (4H, m, arH), 7.50-7.67 (3H, m, arH), $9.53(2 \mathrm{H}, \mathrm{d}, J=12.0 \mathrm{~Hz}, 2 \mathrm{CH}), 11.80(1 \mathrm{H}, \mathrm{s}, \mathrm{CH}) .{ }^{13} \mathrm{C}$ NMR (DMSO- $d_{6}, \delta$ ppm): $11.66\left(\mathrm{CH}_{3}\right), 48.05\left(\mathrm{CH}_{2}\right), 125.43(\mathrm{CH})$, arC: [128.13 (CH), $129.33(\mathrm{CH}), 129.99$ $(\mathrm{CH}), 130.47(\mathrm{CH}), 130.58(\mathrm{CH}), 130.93(\mathrm{CH}), 132.30(\mathrm{CH}), 132.36(\mathrm{CH}), 144.19(\mathrm{C}), 144.52(\mathrm{C})], 143.72$ (CH), $144.37(\mathrm{CH}), 150.46$ (triazole $\mathrm{C}-3), 151.72$ (triazole $\mathrm{C}-5), 194.43(\mathrm{C}=0)$ ). El MS $\mathrm{m} / z(\%)$ : 437.25 ([M + $\left.\mathrm{Na}]^{+}, 100\right), 251.30$ (81), $439.26(69), 447.58$ (62), 360.54 (21), $341.40(20)$.

\section{Gerenal Method for The Synthesis of Compounds 9a,b}

Method 1: A solution of the corresponding compound $8 \mathbf{a}, \mathbf{b}(10 \mathrm{mmol})$ in absolute ethanol $(40 \mathrm{~mL})$ was refluxed in the presence of $\mathrm{NaBH}_{4}(30 \mathrm{mmol})$ for $16 \mathrm{~h}$. After evaporating the solvent under reduced pressure, an oily mass appeared. This was recrystallized from acetone:water (1:3) to afford the desired product.

Method 2: The mixture of the corresponding compound $8 \mathrm{a}, \mathbf{b}(1 \mathrm{mmol})$ and $\mathrm{NaBH}_{4}(3 \mathrm{mmol})$ in ethanol was irradiated in monomode microwave reactor in closed vessel with the pressure control at $125^{\circ} \mathrm{C}$ for 8 min (hold time) at $150 \mathrm{~W}$ maximum power. (The progress of the reaction was monitored by TLC). Then solvent was removed under reduced pressure and a solid appeared. This crude product was washed with water and recrystallized from acetone:water (1:3).

2.1.1.13 2-[2-(4-chlorophenyl)-2-hydroxyethyl]-5-methyl-4-\{(1E,2Z)-3-phenylprop-2-en-1-ylidene]amino\}-2,4dihydro-3H-1,2,4-triazol-3-one (9a) 
Yield: 52\% (Method 1), 85\% (Method 2), m.p: $118-119^{\circ} \mathrm{C}$. FT-IR $\left(\mathrm{U}_{\max } \mathrm{cm}^{-1}\right)$ : $3374(\mathrm{OH}), 3038$ (aromatic $\mathrm{CH}), 1639$ (C=0), 1630 (C=0). ${ }^{1} \mathrm{H}$ NMR (DMSO-d $\left.6, \delta \mathrm{ppm}\right): 2.21\left(3 \mathrm{H}, \mathrm{s}, \mathrm{CH}_{3}\right), 4.84-4.88\left(2 \mathrm{H}, \mathrm{m}, \mathrm{CH}_{2}\right)$, $5.41(1 \mathrm{H}, \mathrm{s}, \mathrm{OH}), 6.25-6.32(2 \mathrm{H}, \mathrm{m}, \mathrm{arH}), 6.39-6.43(1 \mathrm{H}, \mathrm{m}, \mathrm{arH}), 7.01-7.07(1 \mathrm{H}, \mathrm{m}, \mathrm{arH}), 7.23-7.43(5 \mathrm{H}$, $\mathrm{m}, \mathrm{arH}), 7.68(2 \mathrm{H}, \mathrm{d}, J=8.0 \mathrm{~Hz}, 2 \mathrm{CH}), 9.56(1 \mathrm{H}, \mathrm{s}, \mathrm{CH}) .{ }^{13} \mathrm{C} \mathrm{NMR}$ (DMSO-d,$\left.\delta \mathrm{ppm}\right): 11.22\left(\mathrm{CH}_{3}\right), 51.74$ $\left(\mathrm{CH}_{2}\right), 70.02(\mathrm{CH})$, arC: [125.43 (CH), $126.21(\mathrm{CH}), 126.71(\mathrm{CH}), 128.11(\mathrm{CH}), 128.45(\mathrm{CH}), 128.47(\mathrm{CH})$, $128.73(\mathrm{CH}), 128.76(\mathrm{CH}), 129.10(\mathrm{CH}), 132.21(\mathrm{C}), 136.89(\mathrm{C}), 142.20(\mathrm{C})], 129.36(\mathrm{CH}), 133.46(\mathrm{CH})$, 152.78 (triazole C-3), 156.72 (triazole C-5). El MS m/z (\%): $407.22\left([\mathrm{M}+\mathrm{Na}+2]^{+}, 100\right), 409.29$ (56).

\subsubsection{2-[2-(2,4-dichlorophenyl)-2-hydroxyethyl]-5-methyl-4-\{(1E,2Z)-3-phenylprop-2-en-1- ylidene]aminot-2,4-dihydro-3H-1,2,4-triazol-3-one (9b)}

Yield: 50\% (Method 1), 82\% (Method 2), m.p: 100-101 ${ }^{\circ} \mathrm{C}$. FT-IR $\left(\mathrm{U}_{\max }, \mathrm{cm}^{-1}\right)$ ): $3251(\mathrm{OH}), 3038$ (aromatic $\mathrm{CH}), 1689$ (C = 0). ${ }^{1} \mathrm{H}$ NMR (DMSO-d $\left.{ }_{6}, \delta \mathrm{ppm}\right): 2.07\left(3 \mathrm{H}, \mathrm{s}, \mathrm{CH}_{3}\right), 5.21\left(2 \mathrm{H}, \mathrm{s}, \mathrm{CH}_{2}\right), 6.29(1 \mathrm{H}, \mathrm{d}, J=4.0 \mathrm{~Hz}$, $\mathrm{CH}), 6.44(1 \mathrm{H}, \mathrm{d}, J=8.0 \mathrm{~Hz}, \mathrm{OH}), 7.26-7.63(8 \mathrm{H}, \mathrm{m}, \mathrm{arH}), 9.58(3 \mathrm{H}, \mathrm{d}, J=12.0 \mathrm{~Hz}, 3 \mathrm{CH}) .{ }^{13} \mathrm{C}$ NMR (DMSO$\left.d_{6}, \delta \mathrm{ppm}\right): 11.67\left(\mathrm{CH}_{3}\right), 51.78\left(\mathrm{CH}_{2}\right), 70.07(\mathrm{CH}), 125.43(\mathrm{CH}), \operatorname{arC}:[126.70(\mathrm{CH}), 126.70(\mathrm{CH}), 127.96$ $(\mathrm{CH}), 128.04(\mathrm{CH}), 128.72(\mathrm{CH}), 129.07(\mathrm{CH}), 129.34(\mathrm{CH}), 130.00(2 \mathrm{CH}), 139.76(2 \mathrm{C}), 144.52(\mathrm{C}), 144.84$ (C)], $135.85(\mathrm{CH}), 143.73(\mathrm{CH}), 151.72$ (triazole C-3), 156.68 (triazole C-5). El MS m/z (\%): $117.14(100)$, 360.48 (71), 221.01 (62), 155.06 (47), 227.14 (44).

\section{General Method for The Synthesis of Compounds 9a-e}

$\mathrm{NaH}(1 \mathrm{mmol})$ was added the solution of the corresponding compound $9 \mathrm{a}, \mathrm{b}(1 \mathrm{mmol})$ in THF and the mixture was irradiated in monomode microwave reactor in closed vessel with pressure control $100^{\circ} \mathrm{C}$, for 5 min at 150 Watt. Then 4-chlorobenzyl chloride (for 10c and 10e), 2,4-dichlorobenzyl chloride (for 10a and 10d) or 2,6-dichlorobenzyl chloride (for 10b) (3 mmol) was added and MW irradiation was maintained for additional 12 min under the same conditions. After evaporating the solvent under reduced pressure, an oily product appeared. Water was added into it and extracted with $15 \mathrm{~mL}$ of ethyl acetate three times in the presence of $\mathrm{K}_{2} \mathrm{CO}_{3}$. The organic layer was dried on $\mathrm{Na}_{2} \mathrm{SO}_{4}$ and solvent was evaporated under reduced pressure. The crude product was purified by column chromatography on silicagel (nhexane/ethyl acetate, 3:7).

\subsubsection{2-\{2-(4-chlorophenyl)-2-[(2,4-dichlorobenzyl)oxy]ethyl\}-5-methyl-4-\{(1 E,2Z) phenylprop-2-en-1- ylidene]aminot-2,4-dihydro-3H-1,2,4-triazol-3-one (10a)}

Yield: 57\%. FT IR (.max, cm-1): $3061(\mathrm{ar}-\mathrm{CH}), 1681(\mathrm{C}=0), 1557(\mathrm{C}=\mathrm{N}) .1 \mathrm{H}$ NMR (DMSO- $\left.d_{6}, \mathrm{ppm}\right): 2.07$ $\left(3 \mathrm{H}, \mathrm{s}, \mathrm{CH}_{3}\right), 4.90\left(2 \mathrm{H}, \mathrm{s}, \mathrm{CH}_{2}\right), 5.69\left(2 \mathrm{H}, \mathrm{d}, \mathrm{J}=4.0 \mathrm{~Hz}, \mathrm{CH}_{2}\right), 7.31-7.54(12 \mathrm{H}, \mathrm{m}, \mathrm{arH}), 9.53(1 \mathrm{H}, \mathrm{s}, \mathrm{CH}), 9.55$ $(1 \mathrm{H}, \mathrm{s}, \mathrm{CH}), 11.38(1 \mathrm{H}, \mathrm{s}, \mathrm{CH}), 11.82(1 \mathrm{H}, \mathrm{s}, \mathrm{CH}) .13 \mathrm{C}$ NMR (DMSO- $\left.d_{6}, \mathrm{ppm}\right): 11.20\left(\mathrm{CH}_{3}\right), 51.77\left(\mathrm{CH}_{2}\right)$, $52.35\left(\mathrm{CH}_{2}\right), 128.03(\mathrm{CH}), 128.08(\mathrm{CH}), 128.42(\mathrm{CH}), 128.45(\mathrm{CH}), \operatorname{arC}:[130.45(\mathrm{CH}), 131.87(\mathrm{CH}), 13.24$ $(\mathrm{CH}), 133.30(\mathrm{CH}), 133.42(\mathrm{CH}), 133.47(\mathrm{CH}), 133.96(\mathrm{CH}), 135.61(\mathrm{CH}), 136.88(\mathrm{CH}), 142.18(\mathrm{CH}), 143.70$ 
$(\mathrm{CH}), 144.54(\mathrm{CH}), 145.73(\mathrm{C}), 145.97(2 \mathrm{C}), 151.73(\mathrm{C}), 152.81$ (C), $153.44(\mathrm{C})], 154.35$ (triazole C-3), 158.47 (triazole C-5). El MS m/z (\%): 542.18 ([M + 1] $\left.]^{+}, 53\right), 217.18$ (100), 175.21 (71), 161.18 (65).

2.1.1.16 2-\{2-(4-chlorophenyl)-2-[(2,6-dichlorobenzyl)oxy]ethyl\}-5-methyl-4-\{(1 E,2Z)-3-phenylprop-2-en-1ylidene]amino-2,4-dihydro-3H-1,2,4-triazol-3-one (10b)

Yield: 65\%. FT IR ( $\left.\mathrm{U}_{\max } \mathrm{cm}^{-1}\right)$ : 3058 (aromatic $\left.\mathrm{CH}\right), 1688(\mathrm{C}=0), 1581(\mathrm{C}=\mathrm{N}) .{ }^{1} \mathrm{H}$ NMR $\left(\mathrm{DMSO}-d_{6}, \delta\right.$ ppm): $2.07\left(3 \mathrm{H}, \mathrm{s}, \mathrm{CH}_{3}\right), 4.90\left(2 \mathrm{H}, \mathrm{s}, \mathrm{CH}_{2}\right), 5.69\left(2 \mathrm{H}, \mathrm{d}, J=4.0 \mathrm{~Hz}, \mathrm{CH}_{2}\right), 7.31-7.54(12 \mathrm{H}, \mathrm{m}, \mathrm{arH}), 9.53(1 \mathrm{H}$, s, CH), $9.55(1 \mathrm{H}, \mathrm{s}, \mathrm{CH}), 11.38(1 \mathrm{H}, \mathrm{s}, \mathrm{CH}), 11.82(1 \mathrm{H}, \mathrm{s}, \mathrm{CH}) .{ }^{13} \mathrm{C}$ NMR (DMSO- $\left.d_{6}, \delta \mathrm{ppm}\right): 11.20\left(\mathrm{CH}_{3}\right)$, $51.77\left(\mathrm{CH}_{2}\right), 52.35\left(\mathrm{CH}_{2}\right), 128.03(\mathrm{CH}), 128.08(\mathrm{CH}), 128.42(\mathrm{CH}), 128.45(\mathrm{CH}), \operatorname{arC}:[130.45(\mathrm{CH}), 131.87$ $(\mathrm{CH}), 13.24(\mathrm{CH}), 133.30(\mathrm{CH}), 133.42(\mathrm{CH}), 133.47(\mathrm{CH}), 133.96(\mathrm{CH}), 135.61(\mathrm{CH}), 136.88(\mathrm{CH}), 142.18$ $(\mathrm{CH}), 143.70(\mathrm{CH}), 144.54(\mathrm{CH}), 145.73(\mathrm{C}), 145.97$ (C), 151.73 (C), 152.81 (C), 153.44 (C)], 154.35 (triazole C-3), 158.47 (triazole C-5). El MS m/z (\%): 300.55 (100), 287.91 (71), 421.83 (64), $564.87\left([\mathrm{M}+\mathrm{Na}]^{+}, 45\right)$.

2.1.1.17 2-[2-[(2,6-dichlorobenzyl)oxy]-2-(2,4-dichlorophenyl)ethyl]-5-methyl-4-[(1 E,2Z)-3-phenylprop-2-en1-ylidene]aminot-2,4-dihydro-3H-1,2,4-triazol-3-one (10c)

Yield: 58\%. FT IR (.max, cm-1): 3057 (aromatic CH), $1691(\mathrm{C}=0), 1577(\mathrm{C}=\mathrm{N}) .{ }^{1} \mathrm{H}$ NMR (DMSO- $\left.d_{6}, \mathrm{ppm}\right)$ : $1.88\left(3 \mathrm{H}, \mathrm{s}, \mathrm{CH}_{3}\right), 2.41\left(2 \mathrm{H}, \mathrm{s}, \mathrm{CH}_{2}\right), 4.75\left(2 \mathrm{H}, \mathrm{s}, \mathrm{CH}_{2}\right), 5.18(1 \mathrm{H}, \mathrm{s}, \mathrm{CH}), 7.21(3 \mathrm{H}, \mathrm{s}, \mathrm{arH}), 7.47(4 \mathrm{H}, \mathrm{s}, \mathrm{arH})$, 7.72-7.81 (4H, m, arH), $9.81(1 \mathrm{H}, \mathrm{s}, \mathrm{CH}), 11.45(1 \mathrm{H}, \mathrm{s}, \mathrm{CH}), 11.63(1 \mathrm{H}, \mathrm{s}, \mathrm{CH}) .{ }^{13} \mathrm{C}$ NMR (DMSO-d $\left.6, \mathrm{ppm}\right)$ : $11.77\left(\mathrm{CH}_{3}\right), 50.43\left(\mathrm{CH}_{2}\right), 57.10\left(\mathrm{CH}_{2}\right), 121.18(\mathrm{CH}), 122.76(\mathrm{CH}), 123.54(\mathrm{CH}), 126.14(\mathrm{CH}), \operatorname{arC}:[129.02$ $(\mathrm{CH}), 130.04(\mathrm{CH}), 130.76(\mathrm{CH}), 131.13(\mathrm{CH}), 131.84(\mathrm{CH}), 131.90(\mathrm{CH}), 132.00(\mathrm{CH}), 132.14(\mathrm{CH}), 132.22$ $(\mathrm{CH}), 132.41(\mathrm{CH}), 133.52(\mathrm{CH}), 133.76(\mathrm{C}), 134.15(\mathrm{C}), 134.78(\mathrm{C}), 135.17(\mathrm{C}), 135.88(\mathrm{C}), 135.96(\mathrm{C})$, 141.03 (C)], 153.90 (triazole C-3), 157.83 (triazole C-5). El MS m/z (\%): 577.71 ([M + 1]+, 83), 401.43 (52), 322.90 (47), 310.87 (100), 231.62 (34), 110.65 (65).

2.1.1.18 2-[2-[(2,4-dichlorobenzyl)oxy]-2-(2,4-dichlorophenyl)ethyl]-5-methyl-4-\{(1 E,2Z)-3-phenylprop-2-en1-ylidenejaminot-2,4-dihydro-3H-1,2,4-triazol-3-one (10d)

Yield: 93\%. FT IR (.max, cm-1): 3087 (aromatic $\mathrm{CH}), 1698(\mathrm{C}=0), 1561(\mathrm{C}=\mathrm{N}) .{ }^{1} \mathrm{H}$ NMR (DMSO-d $\left.d_{6} . \mathrm{ppm}\right)$ : $2.06\left(3 \mathrm{H}, \mathrm{s}, \mathrm{CH}_{3}\right), 2.23\left(2 \mathrm{H}, \mathrm{s}, \mathrm{CH}_{2}\right), 4.81\left(2 \mathrm{H}, \mathrm{s}, \mathrm{CH}_{2}\right), 5.81(1 \mathrm{H}, \mathrm{d}, J=4.0 \mathrm{~Hz}, \mathrm{CH}), 7.45-7.48(6 \mathrm{H}, \mathrm{m}, \mathrm{arH})$, 7.63-7.69 (5H, m, arH), $9.53(1 \mathrm{H}, \mathrm{d}, J=8.0 \mathrm{~Hz}, \mathrm{CH}), 11.35(1 \mathrm{H}, \mathrm{s}, \mathrm{CH}), 11.81(1 \mathrm{H}, \mathrm{s}, \mathrm{CH}) .{ }^{13} \mathrm{C}$ NMR (DMSO$d_{6}$. ppm): $11.67\left(\mathrm{CH}_{3}\right), 51.77\left(\mathrm{CH}_{2}\right), 56.28\left(\mathrm{CH}_{2}\right), 126.17(\mathrm{CH}), 126.95(\mathrm{CH}), 127.96(\mathrm{CH}), 127.99(\mathrm{CH})$, arC: [128.04 (CH), $128.07(\mathrm{CH}), 128.13(\mathrm{CH}), 128.33(\mathrm{CH}), 128.72(\mathrm{CH}), 129.07(\mathrm{CH}), 129.30(\mathrm{CH}), 129.34(\mathrm{CH})$, $129.35(\mathrm{CH}), 129.44(\mathrm{CH}), 129.68(\mathrm{CH}), 133.02(\mathrm{C}), 133.33(\mathrm{C}), 134.68(\mathrm{C}), 134.73(\mathrm{C}), 135.83(\mathrm{C}), 136.89$ (C), 139.73 (C)], 152.90 (triazole C-3), 156.57 (triazole C-5). El MS m/z (\%): 443.20 (35), 441.12 (48), 413.18 (59), 411.17 (100), 409.16 (60), 284.37 (28).

2.1.1.19 2-[2-[(4-chlorobenzyl)oxy]-2-(2,4-dichlorophenyl)ethyl]-5-methyl-4-[(1 E,2Z)-3-phenylprop-2-en-1ylidenejaminot-2,4-dihydro-3H-1,2,4-triazol-3-one (10e) 
Yield: 87\%. FT IR (.max, cm-1): 3066 (aromatic CH), $1703(\mathrm{C}=0), 1597(\mathrm{C}=\mathrm{N}) .{ }^{1} \mathrm{H}$ NMR (DMSO- $\left.d_{6}, . \mathrm{ppm}\right)$ : $2.09\left(3 \mathrm{H}, \mathrm{s}, \mathrm{CH}_{3}\right), 3.36\left(2 \mathrm{H}, \mathrm{s}, \mathrm{CH}_{2}\right), 3.65-3.68(1 \mathrm{H}, \mathrm{m}, \mathrm{CH}), 4.77\left(2 \mathrm{H}, \mathrm{s}, \mathrm{CH}_{2}\right), 7.25-7.49(12 \mathrm{H}, \mathrm{m}, \mathrm{arH}), 9.48$ $(1 \mathrm{H}, \mathrm{s}, \mathrm{CH}), 9.71(1 \mathrm{H}, \mathrm{s}, \mathrm{CH}), 11.50(1 \mathrm{H}, \mathrm{s}, \mathrm{CH}) .{ }^{13} \mathrm{C} \mathrm{NMR}\left(\mathrm{DMSO}-d_{6}, \mathrm{ppm}\right): 11.21\left(\mathrm{CH}_{3}\right), 45.63\left(\mathrm{CH}_{2}\right), 62.55$ $\left(\mathrm{CH}_{2}\right), 126.20(\mathrm{CH}), 126.70(\mathrm{CH})$, arC: [128.09 (CH), $128.44(\mathrm{CH}), 128.64(\mathrm{CH}), 129.09(\mathrm{CH}), 129.11(\mathrm{CH})$, $129.36(\mathrm{CH}), 130.05(\mathrm{CH}), 130.47(\mathrm{CH}), 131.18(2 \mathrm{C}), 132.20(\mathrm{CH}), 133.45(2 \mathrm{C}), 136.89(2 \mathrm{C}), 137.19(2 \mathrm{C})$, 142.20 (3C)], 144.79 (triazole C-3), 152.78 (triazole C-5). El MS m/z (\%): 542.18 ([M + 1] $\left.]^{+}, 21\right), 432.18(51)$, 411.76 (65), 309.18 (75), 210.87 (100).

\section{GeneralaMethod foraThe Synthesisaof Compounds 11ae}

The solution of norfloxacine (for 11a) $(10 \mathrm{mmol})$ or ciprofloxacine (for $11 \mathrm{~b})(10 \mathrm{mmol})$, or morpholine (for 11c) $(10 \mathrm{mmol})$,aor thiomorpholinea(for $11 \mathrm{~d})(10 \mathrm{mmol})$, or 4-phenylpiperazine (for $11 \mathrm{~d})(10 \mathrm{mmol})$, formaldehyde $(37 \%, 30 \mathrm{mmol})$, product $3(10 \mathrm{mmol})$ aand $\mathrm{HCl}(10 \% \mathrm{~mol})$ wasairradiated in monomode microwaveareactor inaclosed vessel with temperature controlaat $80^{\circ} \mathrm{C}$ fora $5 \mathrm{~min}$ at $100 \mathrm{~W}$. After that resulting solution wasapoured intoaice-water. The precipitated product wasafiltered offaand recrystallizedafrom $\mathrm{DMSO} / \mathrm{H}_{2} \mathrm{O}(1: 3)$ to give the target molecule.

\subsubsection{1-ethyl-6-fluoro-7\{4-[(3-methyl-5-oxo-4-\{(1 E,2Z)-3-phenylprop-2-en-1-ylidene]amino\}-4,5-dihydro- 1H-1,2,4-triazol-1-yl)methyl]piperazin-1-yl\}-4-oxo-1,4-dihydroquinoline-3-carboxylic acid (11a)}

Yield: 67\%, m.p. $121-122^{\circ} \mathrm{C}$. FT IR $\left(\mathrm{U}_{\max } \mathrm{cm}^{-1}\right): 3384(\mathrm{OH}), 3051$ (aromatic $\left.\mathrm{CH}\right), 1715(\mathrm{C}=0), 1624(\mathrm{C}=$ 0), $1479(\mathrm{C}=\mathrm{N}) .{ }^{1} \mathrm{H}$ NMR (DMSO-d $\left.6 . \mathrm{ppm}\right): 1.31\left(3 \mathrm{H}, \mathrm{s}, \mathrm{CH}_{3}\right), 1.39\left(3 \mathrm{H}, \mathrm{s}, \mathrm{CH}_{3}\right), 2.27\left(2 \mathrm{H}, \mathrm{s}, \mathrm{CH}_{2}\right), 2.45(2 \mathrm{H}$, s, $\left.\mathrm{CH}_{2}\right), 2.76\left(2 \mathrm{H}, \mathrm{s}, \mathrm{CH}_{2}\right), 2.83\left(2 \mathrm{H}, \mathrm{s}, \mathrm{CH}_{2}\right), 3.03\left(2 \mathrm{H}, \mathrm{s}, \mathrm{CH}_{2}\right), 4.57\left(2 \mathrm{H}, \mathrm{s}, \mathrm{CH}_{2}\right), 7.33-7.78(7 \mathrm{H}, \mathrm{m}, \mathrm{arH})$, $8.77(2 \mathrm{H}, \mathrm{s}, 2 \mathrm{CH}), 8.93(2 \mathrm{H}, \mathrm{s}, 2 \mathrm{CH}), 15.27(1 \mathrm{H}, \mathrm{s}, \mathrm{OH}) .{ }^{13} \mathrm{C}$ NMR (DMSO-d $\left.d_{6} \mathrm{ppm}\right): 11.53\left(\mathrm{CH}_{3}\right), 18.19$ $\left(\mathrm{CH}_{3}\right), 35.09\left(\mathrm{CH}_{2}\right), 36.71\left(\mathrm{CH}_{2}\right), 37.44\left(\mathrm{CH}_{2}\right), 39.81\left(\mathrm{CH}_{2}\right), 40.90\left(\mathrm{CH}_{2}\right), 47.88\left(\mathrm{CH}_{2}\right), 91.53(\mathrm{CH}), 93.49$ $(\mathrm{CH}), 95.18(\mathrm{CH}), 96.72(\mathrm{CH})$, arC: [120.60 (CH), $121.23(\mathrm{CH}), 122.62(\mathrm{CH}), 122.99(\mathrm{CH}), 123.51(\mathrm{CH})$, $123.91(\mathrm{CH}), 124.59$ (CH), 126.71 (C), 126.92 (C), 128.25 (C), 130.30 (C), 131.51 (C), 133.52 (C)], 156.10 (triazole C-3), 159.41 (triazole $\mathrm{C}-5), 173.87(\mathrm{C}=0), 177.81(\mathrm{C}=0)$. El MS $m / z(\%): 582.27\left([\mathrm{M}+\mathrm{Na}]^{+}, 100\right)$, $560.24\left([\mathrm{M}+1]^{+}, 98\right), 485.17(40), 332.20(31), 354.28(19)$. Elemental analysis for: $\mathrm{C}_{29} \mathrm{H}_{30} \mathrm{FN}_{7} \mathrm{O}_{4}$; Calculated (\%): C, 62.24; H, 5.40; N, 17.52; Found (\%): C, 62.31; H, 5.43; N, 17.60.

\subsubsection{1-cyclopropyl-6-fluoro-7 $\{4-[(3-m e t h y l-5-0 x o-4\{[(1 \mathrm{E}, 2 Z)-3-p h e n y l p r o p-2-e n-1-y l i d e n e] ~ a m i n o\}-4,5-$ dihydro-1H-1,2,4-triazol-1-yl)methyl]piperazin-1-yl\}-4-oxo-1,4-dihydroquinoline-3-carboxylic acid (11b)}

Yield: 63\%, m.p.126-128 C. FT IR $\left(U_{\max }, \mathrm{cm}^{-1}\right): 3416(\mathrm{OH}), 3079$ (aromatic $\left.\mathrm{CH}\right), 1718(\mathrm{C}=0), 1693(\mathrm{C}=$ 0), $1595(\mathrm{C}=\mathrm{N}) .{ }^{1} \mathrm{H}$ NMR (DMSO-d 6 , $\left.\delta \mathrm{ppm}\right): 1.15\left(2 \mathrm{H}, \mathrm{s}, \mathrm{CH}_{2}\right), 1.31\left(2 \mathrm{H}, \mathrm{d}, J=8.0 \mathrm{~Hz}, \mathrm{CH}_{2}\right), 2.25(3 \mathrm{H}, \mathrm{s}$, $\left.\mathrm{CH}_{3}\right), 2.73\left(2 \mathrm{H}, \mathrm{s}, \mathrm{CH}_{2}\right), 2.82\left(2 \mathrm{H}, \mathrm{s}, \mathrm{CH}_{2}\right), 2.89\left(2 \mathrm{H}, \mathrm{s}, \mathrm{CH}_{2}\right), 3.80\left(2 \mathrm{H}, \mathrm{s}, \mathrm{CH}_{2}\right), 4.64\left(2 \mathrm{H}, \mathrm{s}, \mathrm{CH}_{2}\right), 7.29-7.68$ $(6 \mathrm{H}, \mathrm{m}, \mathrm{arH}), 7.82(1 \mathrm{H}, \mathrm{d}, J=12.0 \mathrm{~Hz}, \mathrm{arH}), 8.61(2 \mathrm{H}, \mathrm{s}, 2 \mathrm{CH}), 9.49(2 \mathrm{H}, \mathrm{d}, J=8.0 \mathrm{~Hz}, 2 \mathrm{CH}), 15.17(1 \mathrm{H}, \mathrm{s}$, $\mathrm{OH}) .{ }^{13} \mathrm{C}$ NMR (DMSO-d $\left.d_{6} . \mathrm{ppm}\right): 11.53\left(\mathrm{CH}_{3}\right), 34.10\left(\mathrm{CH}_{2}\right), 36.78\left(\mathrm{CH}_{2}\right), 39.98\left(\mathrm{CH}_{2}\right), 41.40\left(\mathrm{CH}_{2}\right), 42.23$ $\left(\mathrm{CH}_{2}\right), 44.80\left(\mathrm{CH}_{2}\right), 46.71\left(\mathrm{CH}_{2}\right), 90.14(\mathrm{CH}), 91.90(\mathrm{CH}), 93.45(\mathrm{CH}), 94.17(\mathrm{CH}), 96.65(\mathrm{CH})$, arC: [120.78 
$(\mathrm{CH}), 121.65(\mathrm{CH}), 122.79(\mathrm{CH}), 124.61(\mathrm{CH}), 124.78(\mathrm{CH}), 126.34(\mathrm{CH}), 127.28(\mathrm{CH}), 129.87(\mathrm{C}), 130.01$ (C), 130.77 (C), 131.98 (C), 133.50 (C), 136.90 (C)], 153.71 (triazole C-3), 156.02 (triazole C-5), $177.76(\mathrm{C}=$ 0), $179.11(\mathrm{C}=0)$. El MS m/z (\%): $134.92(100), 148.96(59), 152.04$ (50), $188.10(44), 204.08$ (41), $576.39\left([\mathrm{M}+1]^{+}, 36\right), 283.30$ (31). Elemental analysis for: $\mathrm{C}_{30} \mathrm{H}_{30} \mathrm{FN}_{7} \mathrm{O}_{4}$; Calculated (\%): C, 63.04; $\mathrm{H}, 5.29$; $\mathrm{N}, 17.15$; Found (\%): C, 63.10; H, 5.33; N, 17.21.

\subsubsection{5-methyl-2-(morpholin-4-ylmethyl)-4-\{[(1 E,2Z)-3-phenylprop-2-en-1-ylidene]amino\}-2,4-dihydro-3H- 1,2,4-triazol-3-one (11c)}

Yield: 4.45 m.p. $126-128^{\complement} \mathrm{C}$. FT IR $\left(\mathrm{U}_{\mathrm{max}}, \mathrm{cm}^{-1}\right)$ : 3066 (aromatic $\left.\mathrm{CH}\right), 1691(\mathrm{C}=0), 1490(\mathrm{C}=\mathrm{N}) .{ }^{1} \mathrm{H}$ NMR (DMSO-d $\left.d_{6}, \delta \mathrm{ppm}\right): 2.09\left(3 \mathrm{H}, \mathrm{s}, \mathrm{CH}_{3}\right), 2.25\left(2 \mathrm{H}, \mathrm{s}, \mathrm{CH}_{2}\right), 2.57\left(2 \mathrm{H}, \mathrm{d}, J=4.0 \mathrm{~Hz}, \mathrm{CH}_{2}\right), 3.34\left(4 \mathrm{H}, \mathrm{s}, 2 \mathrm{CH}_{2}\right)$, $4.51\left(2 \mathrm{H}, \mathrm{s}, \mathrm{CH}_{2}\right), 7.32-7.44(3 \mathrm{H}, \mathrm{m}, \mathrm{arH}), 7.68$ ve $7.69(2 \mathrm{H}, \mathrm{d}, J=4.0 \mathrm{~Hz}, \mathrm{arH}), 9.50(3 \mathrm{H}, \mathrm{d}, J=12.0 \mathrm{~Hz}$, $3 \mathrm{CH}) .{ }^{13} \mathrm{C} \mathrm{NMR}\left(\mathrm{DMSO}-d_{6}, \delta \mathrm{ppm}\right): 11.57\left(\mathrm{CH}_{3}\right), 50.41\left(\mathrm{CH}_{2}\right), 52.06\left(\mathrm{CH}_{2}\right), 66.31\left(\mathrm{CH}_{2}\right), 66.49\left(2 \mathrm{CH}_{2}\right)$, $125.24(\mathrm{CH}), 128.13(\mathrm{CH}), 129.37(\mathrm{CH})$, arC: [130.12 (CH), $135.78(\mathrm{C}), 144.20(2 \mathrm{CH}), 157.25(2 \mathrm{CH})], 140.38$ (triazole C-3), 150.71 (triazole C-5). El MS $m / z$ (\%): 145.93 (100), $114.20(63), 328.31\left([\mathrm{M}+1]^{+}, 56\right), 213.00$ (50), 382.06 (31), $350.21\left([\mathrm{M}+\mathrm{Na}]^{+}, 15\right)$. Elemental analysis for: $\mathrm{C}_{17} \mathrm{H}_{21} \mathrm{~N}_{5} \mathrm{O}_{2}$; Calculated (\%): $\mathrm{C}, 62.37 ; \mathrm{H}$, 6.47; N, 21.39; Found (\%): C, 62.48; H, 6.57; N, 21.45.

\subsubsection{5-methyl-4-\{[(1 E,2Z)-3-phenylprop-2-en-1-ylidene]amino\}-2-(thiomorpholin-4-ylmethyl)-2,4- dihydro-3H-1,2,4-triazol-3-one (11d)}

Yield: 63\%, m.p. $126-128 \mathrm{C}$. FT IR $\left(\mathrm{U}_{\max } \mathrm{cm}^{-1}\right): 3037$ (aromatic $\left.\mathrm{CH}\right), 1689(\mathrm{C}=\mathrm{O}), 1578(\mathrm{C}=\mathrm{N}) .{ }^{1} \mathrm{H} \mathrm{NMR}$ (DMSO-d $\left.d_{6}, \delta \mathrm{ppm}\right): 1.44\left(3 \mathrm{H}, \mathrm{s}, \mathrm{CH}_{3}\right), 2.09\left(2 \mathrm{H}, \mathrm{s}, \mathrm{CH}_{2}\right), 2.25\left(2 \mathrm{H}, \mathrm{s}, \mathrm{CH}_{2}\right), 2.41\left(2 \mathrm{H}, \mathrm{s}, \mathrm{CH}_{2}\right), 2.89(2 \mathrm{H}, \mathrm{s}$, $\left.\mathrm{CH}_{2}\right), 4.53\left(2 \mathrm{H}, \mathrm{s}, \mathrm{CH}_{2}\right), 7.02-7.08(3 \mathrm{H}, \mathrm{m}, \mathrm{arH}), 7.35(2 \mathrm{H}, \mathrm{s}, \mathrm{arH}), 9.49(2 \mathrm{H}, \mathrm{d}, J=8.0 \mathrm{~Hz}, 2 \mathrm{CH}), 11.74(1 \mathrm{H}, \mathrm{s}$, $\mathrm{CH}) .{ }^{13} \mathrm{C} \mathrm{NMR}\left(\mathrm{DMSO}-d_{6}, \delta \mathrm{ppm}\right): 11.57\left(\mathrm{CH}_{3}\right), 27.62\left(\mathrm{CH}_{2}\right), 51.25\left(\mathrm{CH}_{2}\right), 52.42\left(\mathrm{CH}_{2}\right), 53.39\left(\mathrm{CH}_{2}\right), 62.67$ $\left(\mathrm{CH}_{2}\right), 125.23(\mathrm{CH}), 128.12(\mathrm{CH}), 129.36(\mathrm{CH}), \operatorname{arC}:$ [130.11 (CH), $\left.135.78(\mathrm{C}), 144.19(2 \mathrm{CH}), 157.25(2 \mathrm{CH})\right]$, 143.34 (triazol C-3), 150.65 (triazol C-5). El MS $m / z(\%)$ : 344.78 ([M + 1] $\left.]^{+}, 100\right), 478.14$ (74), 510.51 (66), 123.76 (45). Elemental analysis for: $\mathrm{C}_{17} \mathrm{H}_{21} \mathrm{~N}_{5} \mathrm{OS}$; Calculated (\%): C, 59.45; H, 6.16; N, 20.39; Found (\%): $\mathrm{C}$, $59.80 ; \mathrm{H}, 6.20 ; \mathrm{N}, 20.45$.

\subsubsection{5-methyl-2-[(4-phenylpiperazin-1-yl)methyl]-4-\{(1 E,2Z)-3-phenylprop-2-en-1-ylidene] amino\}-2,4- dihydro-3H-1,2,4-triazol-3-one (11e)}

Yield: 63\%, m.p. $126-128^{\complement} \mathrm{C}$. FT IR $\left(\mathrm{U}_{\mathrm{max}}, \mathrm{cm}^{-1}\right)$ : 3035 (aromatic $\left.\mathrm{CH}\right), 1697(\mathrm{C}=0), 1577(\mathrm{C}=\mathrm{N}) .{ }^{1} \mathrm{H}$ NMR (DMSO-d 6 , $\delta$ ppm): $2.09\left(3 \mathrm{H}, \mathrm{s}, \mathrm{CH}_{3}\right), 2.25\left(2 \mathrm{H}, \mathrm{s}, \mathrm{CH}_{2}\right), 2.59\left(2 \mathrm{H}, \mathrm{s}, \mathrm{CH}_{2}\right), 2.73\left(2 \mathrm{H}, \mathrm{s}, \mathrm{CH}_{2}\right), 3.12(4 \mathrm{H}, \mathrm{s}$, $\left.2 \mathrm{CH}_{2}\right), 4.60\left(2 \mathrm{H}, \mathrm{s}, \mathrm{CH}_{2}\right), 6.77(1 \mathrm{H}, \mathrm{d}, J=4.0 \mathrm{~Hz}, \operatorname{arH}), 6.91(2 \mathrm{H}, \mathrm{d}, J=8.0 \mathrm{~Hz}, \operatorname{arH}), 7.08-7.21(2 \mathrm{H}, \mathrm{m}, \operatorname{arH})$, 7.33-7.44 (3H, m, arH), $7.69(2 \mathrm{H}, \mathrm{d}, J=8.0 \mathrm{~Hz}, \mathrm{arH}), 9.51(3 \mathrm{H}, \mathrm{d}, J=8.0 \mathrm{~Hz}, 3 \mathrm{CH}) .{ }^{13} \mathrm{C}$ NMR (DMSO-d,$\delta$ ppm): $11.58\left(\mathrm{CH}_{3}\right), 48.73\left(\mathrm{CH}_{2}\right), 50.03\left(\mathrm{CH}_{2}\right), 51.50\left(\mathrm{CH}_{2}\right), 66.12\left(2 \mathrm{CH}_{2}\right), 115.86(\mathrm{CH}), 116.09(\mathrm{CH}), 119.39$ $(\mathrm{CH})$, arC: [125.25 (2CH), $128.14(2 \mathrm{CH}), 129.35(2 \mathrm{CH}), 130.13(2 \mathrm{CH}), 135.79(\mathrm{C}), 140.37(\mathrm{C}), 144.21(\mathrm{CH})$, $157.27(\mathrm{CH})], 150.71$ (triazole C-3), 151.52 (triazole C-5). El MS m/z (\%): 403.18 ([M + 1] $\left.]^{+}, 100\right), 256.78$ 
(84), 189.90 (78), 357.81 (65), 288.90 (48). Elemental analysis for: $\mathrm{C}_{23} \mathrm{H}_{26} \mathrm{~N}_{6} \mathrm{O}$; Calculated (\%): C, 68.63; $\mathrm{H}$, $6.51 ; \mathrm{N}, 20.89$; Found (\%): C, 68.71; H, 6.57; N, 20.94.

\subsubsection{5-methyl-4-\{[(1Z,2Z)-3-phenylprop-2-en-1-ylidene]amino\}-2-[(5-sulfanyl-1,3,4-oxadiazol-2- yl)methyl]-2,4-dihydro-3H-1,2,4-triazol-3-one (12)}

$\mathrm{CS}_{2}(10 \mathrm{mmol})$ was added to the solution of compound $5(10 \mathrm{mmol})$ in $50 \mathrm{~mL}$ of ethanol and $50 \mathrm{~mL}$ of $\mathrm{H}_{2} \mathrm{O}$, and the mixture was refluxed in the presence of $\mathrm{KOH}(10 \mathrm{mmol})$ for $10 \mathrm{~h}$. After cooling to room temperature, the mixture was neutralized with $\mathrm{HCl}$. The solid precipitated was collected by filtration and recrystallized from ethanol to give the pure compound.

Yield 63\%, m.p: $121-123^{\circ} \mathrm{C}$. FT IR $\left(\mathrm{U}_{\max } \mathrm{cm}^{-1}\right)$ : 3044 (aromatic $\left.\mathrm{CH}\right), 2749(\mathrm{SH}), 1667(\mathrm{C}=0), 1592(\mathrm{C}=$ N). ${ }^{1} \mathrm{H}$ NMR (DMSO- $\left.d_{6}, \delta \mathrm{ppm}\right): 2.12\left(3 \mathrm{H}, \mathrm{s}, \mathrm{CH}_{3}\right), 5.01\left(2 \mathrm{H}, \mathrm{s}, \mathrm{CH}_{2}\right), 5.32(3 \mathrm{H}, \mathrm{s}, 3 \mathrm{CH}), 7.06-7.12(1 \mathrm{H}, \mathrm{m}$, $\operatorname{arH}), 7.38-7.46(4 \mathrm{H}, \mathrm{m}, \mathrm{arH}), 14.66(1 \mathrm{H}, \mathrm{brs}, \mathrm{SH}) .{ }^{13} \mathrm{C}$ NMR (DMSO- $\left.d_{6}, \delta \mathrm{ppm}\right): 11.58\left(\mathrm{CH}_{3}\right), 56.51\left(\mathrm{CH}_{2}\right)$, $90.10(\mathrm{CH}), 97.45(\mathrm{CH}), 101.30(\mathrm{CH}), \operatorname{arC}:[102.54(\mathrm{CH}), 107.76(\mathrm{CH}), 108.43(\mathrm{CH}), 110.78(\mathrm{CH}), 121.67$ $(\mathrm{CH}), 125.87$ (C)], 150.43 (oxadiazole C-2), 156.89 (oxadiazole C-5), 161.78 (triazole C-3), 167.65 (triazole C-5). EI MS m/z (\%): 251.04 (100), 266.93 (44), $360.41\left(\left[\mathrm{M}+\mathrm{H}_{2} \mathrm{O}\right]^{+}, 38\right), 127.03(19), 381.43\left([\mathrm{M}+\mathrm{K}]^{+}, 13\right)$.

\section{GeneralaMethod foraThe Synthesis of Compounds 13a,b}

The solution of norfloxacine (for 13a) $(10 \mathrm{mmol})$ or ciprofloxacine (for $13 \mathrm{~b})$ (10 mmol), formaldehyde $(37 \%, 30 \mathrm{mmol})$, product $12(10 \mathrm{mmol})$ aand $\mathrm{HCl}(10 \% \mathrm{~mol})$ wasairradiated in monomode microwaveareactor inaclosed vessel with temperature controlaat $80^{\circ} \mathrm{C}$ fora $5 \mathrm{~min}$ at $100 \mathrm{~W}$. After that resulting solution wasapoured intoaice-water. The precipitated product wasafiltered offaand recrystallizedafrom DMSO/ $\mathrm{H}_{2} \mathrm{O}(1: 3)$ to give the target molecule.

\subsubsection{1-ethyl-6-fluoro-7-(4-\{[5-[(3-methyl-5-oxo-4-\{[(1 E,2E)-3-phenylprop-2-en-1-ylidene]amino\}-4,5- dihydro-1H-1,2,4-triazol-1-yl)methyl]-2-thioxo-1,3,4-oxadiazol-3(2H)-yl]methyl\}piperazin-1-yl)-4-oxo-1,4- dihydroquinoline-3-carboxylic acid (13a)}

Yield: 78\%. FT IR ( $\left.U_{\max }, \mathrm{cm}^{-1}\right)$ : $3320(\mathrm{OH}), 3065$ (aromatic $\left.\mathrm{CH}\right), 1720(\mathrm{C}=0), 1698(\mathrm{C}=0), 1597(\mathrm{C}=\mathrm{N})$.

${ }^{1} \mathrm{H}$ NMR (DMSO-d 6 , $\left.\delta \mathrm{ppm}\right): 2.25\left(3 \mathrm{H}, \mathrm{s}, \mathrm{CH}_{3}\right), 2.40\left(3 \mathrm{H}, \mathrm{s}, \mathrm{CH}_{3}\right), 2.65\left(2 \mathrm{H}, \mathrm{s}, \mathrm{CH}_{2}\right), 2.70\left(2 \mathrm{H}, \mathrm{s}, \mathrm{CH}_{2}\right), 2.95$ $\left(2 \mathrm{H}, \mathrm{s}, \mathrm{CH}_{2}\right), 3.78\left(2 \mathrm{H}, \mathrm{s}, \mathrm{CH}_{2}\right), 4.10\left(2 \mathrm{H}, \mathrm{s}, \mathrm{CH}_{2}\right), 4.68\left(2 \mathrm{H}, \mathrm{s}, \mathrm{CH}_{2}\right), 4.80\left(2 \mathrm{H}, \mathrm{s}, \mathrm{CH}_{2}\right), 6.98-7.00(5 \mathrm{H}, \mathrm{m}, \mathrm{arH})$, 7.12-7.40 (2H, m, arH), $8.32(2 \mathrm{H}, \mathrm{s}, 2 \mathrm{CH}), 8.88(1 \mathrm{H}, \mathrm{s}, \mathrm{CH}), 9.12(1 \mathrm{H}, \mathrm{s}, \mathrm{CH}), 15.40(1 \mathrm{H}, \mathrm{s}, \mathrm{OH}) .{ }^{13} \mathrm{C} \mathrm{NMR}$ (DMSO-d 6 , ppm): $11.53\left(\mathrm{CH}_{3}\right), 27.45\left(\mathrm{CH}_{3}\right), 36.09\left(\mathrm{CH}_{2}\right), 38.43\left(\mathrm{CH}_{2}\right), 47.32\left(\mathrm{CH}_{2}\right), 47.90\left(\mathrm{CH}_{2}\right), 48.10$ $\left(\mathrm{CH}_{2}\right), 49.01\left(\mathrm{CH}_{2}\right), 50.43\left(\mathrm{CH}_{2}\right), 91.32(\mathrm{CH}), 91.98(\mathrm{CH}), 92.18(\mathrm{CH}), 96.09(\mathrm{CH}), \operatorname{arC}:[112.32(\mathrm{CH}), 114.56$ $(\mathrm{CH}), 116.87(\mathrm{CH}), 117.43(\mathrm{CH}), 118.18(\mathrm{CH}), 120.41(\mathrm{CH}), 125.87(\mathrm{CH}), 126.65(\mathrm{C}), 130.09(\mathrm{C}), 133.31(\mathrm{C})$, 134.58 (C), 136.76 (C), 137.61 (C)], 150.09 (oxadiazole C-2), 151.78 (oxadiazole C-5), 156. 45 (triazole C3), 158.98 (triazole $C-5), 178.09(C=0), 180.32(C=0)$. El MS m/z (\%): 300.98 (100), $478.23(89), 674.98$ 
$\left([\mathrm{M}+1]^{+}, 78\right), 509.41$ (65), 600.87 (51), 267.47 (38). Elemental analysis for: $\mathrm{C}_{32} \mathrm{H}_{32} \mathrm{FN}_{9} \mathrm{O}_{5} \mathrm{~S}$; Calculated (\%): C, 57.05; H, 4.79; N, 18.71; Found (\%): C, 57.10; H, 4.84; N, 18.77.

\subsubsection{1-cyclopropyl-6-fluoro-7-(4-\{[5-[(3-methyl-5-oxo-4-\{[(1 E,2E)-3-phenylprop-2-en-1-} ylidene]amino\}-4,5-dihydro-1H-1,2,4-triazol-1-yl)methyl]-2-thioxo-1,3,4-oxadiazol-3(2H)-yl]methyl\}piperazin1-yl)-4-oxo-1,4-dihydroquinoline-3-carboxylic acid (13b)

Yield: 75\%. FT IR $\left(U_{\text {max }}, \mathrm{cm}^{-1}\right)$ : $3412(\mathrm{OH}), 3067$ (aromatic $\left.\mathrm{CH}\right), 1722(\mathrm{C}=0), 1696(\mathrm{C}=0), 1587(\mathrm{C}=\mathrm{N})$. ${ }^{1} \mathrm{H}$ NMR (DMSO-d $\left.d_{6}, \delta \mathrm{ppm}\right): 1.30\left(2 \mathrm{H}, \mathrm{s}, \mathrm{CH}_{2}\right), 1.45\left(2 \mathrm{H}, \mathrm{s}, \mathrm{CH}_{2}\right), 2.41\left(3 \mathrm{H}, \mathrm{s}, \mathrm{CH}_{3}\right), 2.65\left(2 \mathrm{H}, \mathrm{s}, \mathrm{CH}_{2}\right), 2.71$ $\left(2 \mathrm{H}, \mathrm{s}, \mathrm{CH}_{2}\right), 2.88\left(2 \mathrm{H}, \mathrm{s}, \mathrm{CH}_{2}\right), 3.76\left(2 \mathrm{H}, \mathrm{s}, \mathrm{CH}_{2}\right), 4.90\left(2 \mathrm{H}, \mathrm{s}, \mathrm{CH}_{2}\right), 5.11\left(2 \mathrm{H}, \mathrm{s}, \mathrm{CH}_{2}\right), 6.98-7.12(6 \mathrm{H}, \mathrm{m}$, arH), 7.15-7.20 (1H, m, arH), $8.10(1 \mathrm{H}, \mathrm{s}, \mathrm{CH}), 8.56(1 \mathrm{H}, \mathrm{s}, \mathrm{CH}), 9.27(1 \mathrm{H}, \mathrm{s}, \mathrm{CH}), 9.77(2 \mathrm{H}, \mathrm{s}, 2 \mathrm{CH}), 15.56$ $(1 \mathrm{H}, \mathrm{s}, \mathrm{OH}) .{ }^{13} \mathrm{C}$ NMR (DMSO-d, $\left.\mathrm{ppm}\right): 12.67\left(\mathrm{CH}_{3}\right), 33.98\left(\mathrm{CH}_{2}\right), 35.61\left(\mathrm{CH}_{2}\right), 37.09\left(\mathrm{CH}_{2}\right), 46.32\left(\mathrm{CH}_{2}\right)$, $47.90\left(\mathrm{CH}_{2}\right), 48.65\left(\mathrm{CH}_{2}\right), 49.31\left(\mathrm{CH}_{2}\right), 50.12\left(\mathrm{CH}_{2}\right), 91.08(\mathrm{CH}), 92.13(\mathrm{CH}), 93.51(\mathrm{CH}), 94.65(\mathrm{CH}), 95.87$ $(\mathrm{CH})$, arC: $[112.05(\mathrm{CH}), 113.56(\mathrm{CH}), 117.71(\mathrm{CH}), 118.54(\mathrm{CH}), 120.41(\mathrm{CH}), 123.76(\mathrm{CH}), 125.61(\mathrm{CH})$, 130.78 (C), 131.90 (C), 132.46 (C), 133.51 (C), 135.09 (C), 136.87 (C)], 148.89 (oxadiazole C-2), 150.76 (oxadiazole $\mathrm{C}-5$ ), 153.90 (triazole $\mathrm{C}-3), 158.41$ (triazole $\mathrm{C}-5), 178.80(\mathrm{C}=0), 180.65(\mathrm{C}=0)$ ). El MS $\mathrm{m} / \mathrm{z}(\%)$ : 389.76 (100), 177.47 (89), $686.65\left([\mathrm{M}+1]^{+}, 80\right), 471.98$ (73), 512.67 (56), 300.54 (43). Elemental analysis for: $\mathrm{C}_{33} \mathrm{H}_{32} \mathrm{FN}_{9} \mathrm{O}_{5} \mathrm{~S}$; Calculated (\%): C, 57.80; H, 4.70; N, 18.38; Found (\%): C, 57.86; H, 4.76; N, 18.43.

\section{General Method for The Synthesis of Compounds 14a-e}

The mixture of norfloxacine (for 14a and 14b) or ciprofloxacine (for 14c, 14d, 14e) (1 mmol) formaldehyde ( $37 \%, 3 \mathrm{mmol})$, compound $7 \mathrm{a}-\mathrm{c}(1 \mathrm{mmol})$ and $\mathrm{HCl}(10 \% \mathrm{~mol})$ was irradiated in monomode microwave reactor in closed vessel with pressure control at $80^{\circ} \mathrm{C}$ for $5 \mathrm{~min}$ at $100 \mathrm{~W}$ (the progress of the reaction was monitored by TLC). Then the resulting solution was poured into ice-water. The product precipitated was filtered off and recrystallized from $\mathrm{DMSO} / \mathrm{H}_{2} \mathrm{O}(1: 3)$ to give the, desired compound.

\subsubsection{1-ethyl-6-fluoro-7-[4-(\{3-[(3-methyl-5-oxo-4-\{[(1 E,2Z)-3-phenylprop-2-en-1-ylidene] amino\}-4,5- dihydro-1H-1,2,4-triazol-1-yl)methyl]-4-phenyl-5-thioxo-4,5-dihydro-1H-1,2,4-triazol-1-yl\}methyl)piperazin-1- yl]-4-oxo-1,4-dihydroquinoline-3-carboxylicacid}

(14a)

Yield: 67\%, m.p. $121-122^{\circ} \mathrm{C}$. FT IR (.max, cm-1): 3059 (aromatic CH), 1710, 1669 and $1626(3 \mathrm{C}=0)$, $1498(\mathrm{C}=\mathrm{N}) .{ }^{1} \mathrm{H}$ NMR (DMSO-d 6 , ppm): $1.42\left(3 \mathrm{H}, \mathrm{s}, \mathrm{CH}_{3}\right), 2.12\left(3 \mathrm{H}, \mathrm{s}, \mathrm{CH}_{3}\right), 2.73\left(2 \mathrm{H}, \mathrm{s}, \mathrm{CH}_{2}\right), 2.89(2 \mathrm{H}, \mathrm{s}$, $\left.\mathrm{CH}_{2}\right), 2.98\left(2 \mathrm{H}, \mathrm{s}, \mathrm{CH}_{2}\right), 4.59\left(4 \mathrm{H}, \mathrm{s}, 2 \mathrm{CH}_{2}\right), 4.97\left(2 \mathrm{H}, \mathrm{s}, \mathrm{CH}_{2}\right), 5.22\left(2 \mathrm{H}, \mathrm{s}, \mathrm{CH}_{2}\right), 7.19-7.78(9 \mathrm{H}, \mathrm{m}, \mathrm{arH})$, 7.89-7.95 (3H, m, arH), $8.94(2 \mathrm{H}, \mathrm{d}, J=12.0 \mathrm{~Hz}, 2 \mathrm{CH}), 9.09(1 \mathrm{H}, \mathrm{d}, J=8.0 \mathrm{~Hz}, \mathrm{CH}), 9.31(1 \mathrm{H}, \mathrm{s}, \mathrm{CH}), 15.36$ $(1 \mathrm{H}, \mathrm{s}, \mathrm{OH}) .{ }^{13} \mathrm{C}$ NMR $\left(\mathrm{DMSO}-d_{6}, \mathrm{ppm}\right): 11.31\left(\mathrm{CH}_{3}\right), 14.81\left(\mathrm{CH}_{3}\right), 36.24\left(\mathrm{CH}_{2}\right), 49.54\left(2 \mathrm{CH}_{2}\right), 50.06\left(2 \mathrm{CH}_{2}\right)$, $66.23\left(2 \mathrm{CH}_{2}\right), 106.51(\mathrm{CH}), 107.55(\mathrm{C}), 111.74(2 \mathrm{CH}), \operatorname{arC}$ : [119.84 (C), $127.54(2 \mathrm{CH}), 128.10(2 \mathrm{CH}), 129.31$ $(2 \mathrm{CH}), 129.47(3 \mathrm{CH}), 129.70(3 \mathrm{CH}), 133.81(\mathrm{C}), 137.64(2 \mathrm{C}), 146.89(3 \mathrm{C})], 157.12$ (triazole 2C-3), 166.58 (triazole 2C-5), $172.15(\mathrm{C}=0), 176.64(\mathrm{C}=0)$. El MS m/z (\%): $749.61([\mathrm{M}+1]+, 63), 507.83(100), 498.15$ 
(50), 387.76 (69), 311.45 (77), 228.71 (37), 178.90 (41). Elemental analysis for: $\mathrm{C}_{38} \mathrm{H}_{37} \mathrm{FN}_{10} \mathrm{O}_{4} \mathrm{~S}$; Calculated (\%): C, 60.95; H, 4.98; N, 18.70; Found (\%): C, 70.00; H, 5.05; N, 18.78.

2.1.1.29 1-ethyl-6-fluoro-7-[4-(\{3-[(3-methyl-5-oxo-4-\{[(1E,2E)-3-phenylprop-2-en-1-yli dene] amino\}-4,5dihydro-1H-1,2,4-triazol-1-yl)methyl]-4-benzyl-5-thioxo-4,5-dihydro-1H-1,2,4-triazol-1-yl\}methyl)piperazin-1yl]-4-oxo-1,4-dihydroquinoline-3-carboxylic acid (14b)

Yield: 75\%, m.p.131-133ㄷ. FT IR $\left(U_{\max } \mathrm{cm}^{-1}\right)$ : $3210(\mathrm{OH}), 3057$ (aromatic $\left.\mathrm{CH}\right), 1695(\mathrm{C}=0), 1681(\mathrm{C}=$ 0), $1507(\mathrm{C}=\mathrm{N}) .{ }^{1} \mathrm{H}$ NMR (DMSO- $\left.d_{6}, \delta \mathrm{ppm}\right): 1,40\left(3 \mathrm{H}, \mathrm{s}, \mathrm{CH}_{3}\right), 2,14\left(3 \mathrm{H}, \mathrm{s}, \mathrm{CH}_{3}\right), 2.19\left(2 \mathrm{H}, \mathrm{s}, \mathrm{CH}_{2}\right), 2.73$ $\left(2 \mathrm{H}, \mathrm{s}, \mathrm{CH}_{2}\right), 2,89\left(2 \mathrm{H}, \mathrm{s}, \mathrm{CH}_{2}\right), 2,98\left(2 \mathrm{H}, \mathrm{s}, \mathrm{CH}_{2}\right), 4.60\left(4 \mathrm{H}, \mathrm{s}, 2 \mathrm{CH}_{2}\right), 4.95\left(2 \mathrm{H}, \mathrm{s}, \mathrm{CH}_{2}\right), 5,20\left(2 \mathrm{H}, \mathrm{s}, \mathrm{CH}_{2}\right)$, 7.23-7.47 (6H, m, arH), 7,63 (6H, m, arH), $7.89(1 \mathrm{H}, \mathrm{s}, \mathrm{arH}), 8,93(1 \mathrm{H}, \mathrm{s}, \mathrm{arH}), 8.96(2 \mathrm{H}, \mathrm{s}, 2 \mathrm{CH}), 9.31(1 \mathrm{H}, \mathrm{s}$, $\mathrm{CH}), 15.36(1 \mathrm{H}, \mathrm{s}, \mathrm{OH}) .{ }^{13} \mathrm{C} \mathrm{NMR}\left(\mathrm{DMSO}-d_{6}, \delta \mathrm{ppm}\right): 12,20\left(\mathrm{CH}_{3}\right), 25,65\left(\mathrm{CH}_{3}\right), 30,65\left(\mathrm{CH}_{2}\right), 31,84\left(\mathrm{CH}_{2}\right)$, $35.41\left(\mathrm{CH}_{2}\right), 38.74\left(\mathrm{CH}_{2}\right), 39.43\left(\mathrm{CH}_{2}\right), 40.65\left(\mathrm{CH}_{2}\right), 41.52\left(\mathrm{CH}_{2}\right), 48.20\left(\mathrm{CH}_{2}\right), 98.01(\mathrm{CH}), 98.86(\mathrm{CH})$, $99.12(\mathrm{CH}), 100,06(\mathrm{CH})$, arC: [111.87 and $111.98(\mathrm{CH}, \mathrm{d}, J=11.0 \mathrm{~Hz}), 112.20(\mathrm{CH}), 112.95117 .20(\mathrm{CH})$, $119.30(\mathrm{CH}), 132.52(\mathrm{CH}), 133.57(\mathrm{CH}), 135.18$ and $134.34(\mathrm{CH}, \mathrm{d}, J=16.0 \mathrm{~Hz}), 136.10(\mathrm{CH}), 137.32(\mathrm{CH})$, $138.10(\mathrm{CH}), 139.12(\mathrm{CH}), 139.69(\mathrm{CH}), 140.02$ (C), 140.49 (C), 141.05 (C), 141.94 (C), 142.01 (C), 142.10 and $142.65(\mathrm{C}, \mathrm{d}, J=55.0 \mathrm{~Hz}$ )], 153.54 (triazole C-3), 154.01 (triazole C-3), 160.71 (triazole C-5), 166.09 (triazole $\mathrm{C}-5), 171.10(\mathrm{C}=0), 177.73(\mathrm{C}=0)$. El MS $m / z(\%): 763.85\left([\mathrm{M}+1]^{+}, 100\right), 507.83(63), 498.90$ (50), 118.80 (37). Elemental analysis for: $\mathrm{C}_{39} \mathrm{H}_{39} \mathrm{FN}_{10} \mathrm{O}_{4} \mathrm{~S}$; Calculated (\%), C, 61.40; $\mathrm{H}, 5.15 ; \mathrm{N}, 18.36$; Found (\%): C, 61.38; H, 5.14; N, 18.35 .

2.1.1.30 1-cyclopropyl-6-fluoro-7-[4-(\{3-[(3-methyl-5-oxo-4-\{[(1E,2Z)-3-phenylprop-2-en-1-ylidene]amino\}-4,5dihydro-1 H-1,2,4-triazol-1-yl)methyl]-5-oxo-4-phenyl-4,5-dihydro-1H-1,2,4-triazol-1-yl\}methyl)piperazin-1yl]-4-oxo-1,4-dihydroquinoline-3-carboxylic acid (14c)

Yield: 63\%, m.p.126-128 C. FT IR $\left(\mathrm{U}_{\max } \mathrm{cm}^{-1}\right): 3290(\mathrm{OH}), 3063$ (aromatic $\left.\mathrm{CH}\right), 1689(\mathrm{C}=0), 1626(\mathrm{C}=$ 0), $1534(\mathrm{C}=\mathrm{N}) .{ }^{1} \mathrm{H}$ NMR (DMSO-d $\left.d_{6}, \delta \mathrm{ppm}\right): 1.18\left(2 \mathrm{H}, \mathrm{s}, \mathrm{CH}_{2}\right), 1.31\left(2 \mathrm{H}, \mathrm{s}, \mathrm{CH}_{2}\right), 2.25\left(3 \mathrm{H}, \mathrm{s}, \mathrm{CH}_{3}\right), 2.73-$ $2.89\left(4 \mathrm{H}, \mathrm{s}, 2 \mathrm{CH}_{2}\right), 3.82\left(2 \mathrm{H}, \mathrm{s}, \mathrm{CH}_{2}\right), 4.14\left(2 \mathrm{H}, \mathrm{s}, \mathrm{CH}_{2}\right), 4.45\left(2 \mathrm{H}, \mathrm{s}, \mathrm{CH}_{2}\right), 4.50\left(2 \mathrm{H}, \mathrm{s}, \mathrm{CH}_{2}\right), 7.04(2 \mathrm{H}, \mathrm{s}, \mathrm{arH})$, 7.29-7.55 (8H, m, arH), $7.69(1 \mathrm{H}, \mathrm{s}, \mathrm{arH}), 7.89(1 \mathrm{H}, \mathrm{s}, \operatorname{arH}), 8.66(2 \mathrm{H}, \mathrm{s}, 2 \mathrm{CH}), 9.19(1 \mathrm{H}, \mathrm{s}, \mathrm{CH}), 9.48(1 \mathrm{H}, \mathrm{s}$, $\mathrm{CH}), 10.04(1 \mathrm{H}, \mathrm{s}, \mathrm{CH}), 15.23(1 \mathrm{H}, \mathrm{s}, \mathrm{OH}) .{ }^{13} \mathrm{C} \mathrm{NMR}\left(\mathrm{DMSO}-d_{6}, \delta \mathrm{ppm}\right): 11.34\left(\mathrm{CH}_{3}\right), 30.19\left(\mathrm{CH}_{2}\right), 31.21$ $\left(\mathrm{CH}_{2}\right)$, $33.10\left(\mathrm{CH}_{2}\right), 33.95\left(\mathrm{CH}_{2}\right), 34.41\left(\mathrm{CH}_{2}\right), 36.89\left(\mathrm{CH}_{2}\right), 39.71\left(\mathrm{CH}_{2}\right), 46.53\left(\mathrm{CH}_{2}\right), 96.12(\mathrm{CH}), 97.61$ (CH), $98.54(\mathrm{CH}), 99.18(\mathrm{CH}), 99.89(\mathrm{CH})$, arC: [110.87 and $110.98(\mathrm{CH}, \mathrm{d}, J=11.0 \mathrm{~Hz}), 111.90(\mathrm{CH}), 112.45$ $(\mathrm{CH}), 113.76(\mathrm{CH}), 121.17(\mathrm{CH}), 124.90(\mathrm{CH}), 126.78(\mathrm{CH}), 129.67(\mathrm{CH}), 130.43(\mathrm{CH}), 131.81(\mathrm{CH}), 132.64$ $(\mathrm{CH}), 134.18$ and $134.34(\mathrm{CH}, \mathrm{d}, J=16.0 \mathrm{~Hz}), 135.87(\mathrm{C}), 136.71(\mathrm{C}), 137.63(\mathrm{C}), 137.90(\mathrm{C}), 138.01(\mathrm{C})$, $139.95(C), 140.10$ and $140.65(C, d, J=55.0 \mathrm{~Hz}$ )], 153.33 (triazole $C-3), 154.10$ (triazole C-3), 160.78 (triazole C-5), 166.94 (triazole $\mathrm{C}-5), 170.19(\mathrm{C}=0), 178.76(\mathrm{C}=0)$. El MS $m / z(\%): 745.78\left([\mathrm{M}+1]^{+}, 67\right)$, 543.65 (73), 498. 90 (70), 300.89 (100), 110.90 (43). Elemental analysis for: $\mathrm{C}_{39} \mathrm{H}_{37} \mathrm{FN}_{10} \mathrm{O}_{5}$; Calculated (\%), C, 62.89; H, 5.01; N, 18.81; Found (\%): C, 62.93; H, 5.09; N, 18.90. 
2.1.1.31 1-cyclopropyl-6-fluoro-7-[4-(\{3-[(3-methyl-5-oxo-4-\{[(1E,2Z)-3-phenylprop-2-en-1-ylidene]amino\}-4,5dihydro-1 H-1,2,4-triazol-1-yl)methyl]-4-phenyl-5-thioxo-4,5-dihydro-1H-1,2,4-triazol-1-yl\}methyl)piperazin-1yl]-4-oxo-1,4-dihydroquinoline-3-carboxylic acid (14d)

Yield: 63\%, m.p. 126-128 C. FT IR (.max, cm-1): 3059 (aromatic CH), 1711, 1670 and $1626(3 \mathrm{C}=0), 1546$ $(\mathrm{C}=\mathrm{N}) .{ }^{1} \mathrm{H}$ NMR $\left(\mathrm{DMSO}-d_{6}, \mathrm{ppm}\right): 1.06\left(3 \mathrm{H}, \mathrm{s}, \mathrm{CH}_{3}\right), 1.16\left(2 \mathrm{H}, \mathrm{s}, \mathrm{CH}_{2}\right), 1.32\left(2 \mathrm{H}, \mathrm{s}, \mathrm{CH}_{2}\right), 2.11(2 \mathrm{H}, \mathrm{d}, J=8.0$ $\left.\mathrm{Hz}, \mathrm{CH}_{2}\right), 2.73\left(2 \mathrm{H}, \mathrm{s}, \mathrm{CH}_{2}\right), 2.89\left(2 \mathrm{H}, \mathrm{s}, \mathrm{CH}_{2}\right), 2.98\left(2 \mathrm{H}, \mathrm{s}, \mathrm{CH}_{2}\right), 4.97\left(2 \mathrm{H}, \mathrm{s}, \mathrm{CH}_{2}\right), 5.22\left(2 \mathrm{H}, \mathrm{s}, \mathrm{CH}_{2}\right), 7.26-$ $7.47(10 \mathrm{H}, \mathrm{m}, \mathrm{arH}), 7.62(2 \mathrm{H}, \mathrm{m}, \mathrm{arH}), 7.95(1 \mathrm{H}, \mathrm{s}, \mathrm{CH}), 8.63(2 \mathrm{H}, \mathrm{d}, J=16.0 \mathrm{~Hz}, 2 \mathrm{CH}), 9.08(1 \mathrm{H}, \mathrm{d}, J=12.0$ $\mathrm{Hz}, \mathrm{CH}), 9.30(1 \mathrm{H}, \mathrm{s}, \mathrm{CH}), 15.20(1 \mathrm{H}, \mathrm{s}, \mathrm{OH}) .{ }^{13} \mathrm{C} \mathrm{NMR}\left(\mathrm{DMSO}-d_{6} . \mathrm{ppm}\right): 8.05\left(\mathrm{CH}_{3}\right), 36.24\left(2 \mathrm{CH}_{2}\right), 49.88$ $\left(2 \mathrm{CH}_{2}\right), 50.01\left(2 \mathrm{CH}_{2}\right), 69.08\left(2 \mathrm{CH}_{2}\right), 106.97(\mathrm{CH}), 111.26(\mathrm{CH}), 111.49(\mathrm{CH}), 119.07(\mathrm{CH}), 119.14(\mathrm{CH}), \operatorname{arC}$ : [124.87 (CH), $127.96(\mathrm{CH}), 128.07(\mathrm{CH}), 128.19(\mathrm{CH}), 129.30(\mathrm{CH}), 129.36(\mathrm{CH}), 129.46(\mathrm{CH}), 129.71(\mathrm{CH})$, $132.13(\mathrm{CH}), 133.50$ (C), 133.80 (C), 135.61 (C), 136.57 (C), 139.75 (C), 144.28 and 144.36 (C, d, J= 8.0 $\mathrm{Hz}$ ), $146.90(\mathrm{C}), 149.01(\mathrm{CH}), 152.23(\mathrm{CH})], 148.35$ (quinolon $\mathrm{CH}$ ), 154.62 (triazole C-3), 154.70 (triazole C3), 157.09 (triazole $C-5), 162.78$ (triazole $C-5), 169.98(C=0), 176.78(C=0)$. El MS m/z (\%): 761.81 ([M + $\left.1]^{+}, 53\right), 507.21$ (89), 451.76 (100), 443.43 (71), 410.65 (39), 127.78 (22). Elemental analysis for: $\mathrm{C}_{39} \mathrm{H}_{37} \mathrm{FN}_{10} \mathrm{O}_{4} \mathrm{~S}$ için; Calculated (\%): C, 61.57; H, 4.90; N, 18.41; Found (\%): C, 61.61; H, 4.96; N, 18.50.

\subsubsection{1-cyclopropyl-6-fluoro-7-[4-(\{3-[(3-methyl-5-oxo-4-\{(1E,2E)-3-phenylprop-2-en-1-yli dene]amino-4,5-dihydro-1 H-1,2,4-triazol-1-yl)methyl]-4-benzyl-5-thioxo-4,5-dihydro-1H-1,2,4-triazol-1- yl\}methyl)piperazin-1-yl]-4-oxo-1,4-dihydroquinoline-3-carboxylic acid (14e)}

Yield: 71\%, m.p.129-131 ${ }^{\square}$ C. FT IR $\left(U_{\max }, \mathrm{cm}^{-1}\right)$ : $3217(\mathrm{OH}), 3061$ (aromatic $\left.\mathrm{CH}\right), 1678(\mathrm{C}=0), 1703(\mathrm{C}=$ 0), $1523(\mathrm{C}=\mathrm{N}) .{ }^{1} \mathrm{H}$ NMR (DMSO- $\left.d_{6}, \delta \mathrm{ppm}\right): 1,75\left(3 \mathrm{H}, \mathrm{s}, \mathrm{CH}_{3}\right), 2.12\left(2 \mathrm{H}, \mathrm{s}, \mathrm{CH}_{2}\right), 2.65\left(2 \mathrm{H}, \mathrm{s}, \mathrm{CH}_{2}\right), 2,85$ $\left(2 \mathrm{H}, \mathrm{s}, \mathrm{CH}_{2}\right), 2,97\left(2 \mathrm{H}, \mathrm{s}, \mathrm{CH}_{2}\right), 4.33\left(2 \mathrm{H}, \mathrm{s}, \mathrm{CH}_{2}\right), 4.78\left(4 \mathrm{H}, \mathrm{s}, 2 \mathrm{CH}_{2}\right), 5.05\left(2 \mathrm{H}, \mathrm{s}, \mathrm{CH}_{2}\right), 5.29\left(2 \mathrm{H}, \mathrm{s}, \mathrm{CH}_{2}\right)$, 7.10-7.39 (8H, m, arH), 7,45-7.69 (4H, m, arH), 8,87 (1H, s, CH), $8.94(1 \mathrm{H}, \mathrm{s}, \mathrm{CH}), 9.12(1 \mathrm{H}, \mathrm{s}, \mathrm{CH}), 9.38(1 \mathrm{H}$, $\mathrm{s}, \mathrm{CH}), 15.40(1 \mathrm{H}, \mathrm{s}, \mathrm{OH}) .{ }^{13} \mathrm{C} \mathrm{NMR}\left(\mathrm{DMSO}-d_{6}, \delta \mathrm{ppm}\right): 11.70\left(\mathrm{CH}_{3}\right), 30.18\left(\mathrm{CH}_{2}\right), 31.87\left(\mathrm{CH}_{2}\right), 36.41$ $\left(2 \mathrm{CH}_{2}\right), 38.54\left(\mathrm{CH}_{2}\right), 39.17\left(\mathrm{CH}_{2}\right), 47.10\left(\mathrm{CH}_{2}\right), 48.19\left(\mathrm{CH}_{2}\right), 49.13\left(\mathrm{CH}_{2}\right), 98.15(\mathrm{CH}), 98.40(\mathrm{CH}), 99.06$ $(\mathrm{CH}), 99.84(\mathrm{CH}), 100.01(\mathrm{CH})$, arC: [110.87 and $110.98(\mathrm{CH}, \mathrm{d}, J=11.0 \mathrm{~Hz}), 111.85(\mathrm{CH}), 112.78113 .05$ $(\mathrm{CH}), 114.52(\mathrm{CH}), 115.47(\mathrm{CH}), 116.73(\mathrm{CH}), 123.10$ and $123.45(\mathrm{CH}, \mathrm{d}, J=35.0 \mathrm{~Hz}), 127.52(\mathrm{CH}), 129.41$ $(\mathrm{CH}), 130.59(\mathrm{CH}), 131.84(\mathrm{CH}), 132.43(\mathrm{CH}), 139.20(\mathrm{C}), 140.45(\mathrm{C}), 141.37(\mathrm{C}), 142.73(\mathrm{C}), 143.11(\mathrm{C})$, $143.79(\mathrm{C}), 144.10$ and $144.65(\mathrm{C}, \mathrm{d}, J=55.0 \mathrm{~Hz}$ )], 155.41 (triazole $\mathrm{C}-3), 158.43$ (triazole C-3), 161.08 (triazole C-5), 165.49 (triazole C-5), $170.21(\mathrm{C}=0), 173.23(\mathrm{C}=0)$. El MS $m / z(\%): 797.29\left([\mathrm{M}+\mathrm{Na}]^{+}, 67\right)$, 774.29 ([M] $\left.]^{+}, 90\right), 543.63$ (53), 490. 90 (40). Elemental analysis: for $\mathrm{C}_{40} \mathrm{H}_{39} \mathrm{FN}_{10} \mathrm{O}_{4}$ S; Calculated (\%), C, $62.00 ; \mathrm{H}, 5.07 ; \mathrm{N}, 18.08$; Found (\%): C, 62.01; H, 5.09; N, 18.06 .

\subsection{The Determination of Antimicrobial Activity}

All bacterial and yeasts were acquired from the Hifzissihha Institute of Refik Saydam (Ankara, Turkey) and were as follows: "Escherichia coli ATCC35218, Yersinia pseudotuberculosis ATCC911, Pseudomonas aeruginosa ATCC 27853, Staphylococcus aureus ATCC 25923, Enterococcus faecalis ATCC 29212, 
Bacillus cereus 709 ROMA, Mycobacterium smegmatis ATCC607, Candida albicans ATCC 60193, Saccharomyces cerevisiae RSKK 251". Novel obtained hybrid molecules were weighed and dissolved in hexane to prepare extract stock solution of 20.000 microgram $/ \mathrm{milliliter}(\mu \mathrm{g} / \mathrm{mL})$.

Antibacterial impacts of the products were tested quantitatively in respective broth mediaaby utilizing double microdilution and the minimal inhibition concentration (MIC) values $(\mu \mathrm{g} / \mathrm{mL})$ were obtained. The antimicrobial and antifungal processes were afforded in Mueller-Hinton broth (MH) (Difco, Detroit, $\mathrm{MI})$ at pH.7.3 and buffered Yeast Nitrogen Base (Difco, Detroit, MI) at pH.7.3, respectively. The micro dilution trial plaques were incubated for $18-24 \mathrm{~h}$ at $35^{\circ} \mathrm{C}$. Brain Heart Infusion broth (BHI) (Difco, Detroit, MI) was utilized for M.asmegmatis, and waited for $48-72 \mathrm{~h}$ at $35^{\circ} \mathrm{C}$ [35]. Ampicillin (10 $\left.\mu \mathrm{g}\right)$, Streptomisin (10 $\left.\mu \mathrm{g}\right)$, and fluconazole $(5 \mu \mathrm{g})$ were utilized as standard antimicrobial and antifungal medicines, respectively. Dimethylsulphoxide with dilution of 1:10 was utilized as solvent check.

\subsection{Anticancer Activity}

\subsubsection{Cell Culture and Cell Proliferation Tests 2.3.1.1 Cell Culture}

All the antiproliferative activity studies were performed in the sterile cabinet. The used medium (DMEM) in sterile culture flasks was transferred to the waste container. $10 \mathrm{~mL}$ of trypsin-EDTA was added to the culture flask containing the cells. The flask's lid was closed and incubated for $1-2$ minutes at $37^{\circ} \mathrm{C}$ in a $\mathrm{CO}_{2}$ incubator $\left(5 \% \mathrm{CO}_{2}\right)$. Thus, the cells adhering to the surface were removed from the surface. After incubation, the medium was neutralized by adding $10 \mathrm{~mL}$ of media (DMEM) to the container. The cell suspension was transferred in equal amounts to two separate $15-\mathrm{mL}$ falcon tubes. These tubes were centrifuged at $600 \mathrm{rpm}$ for 5 minutes to allow the cells to settle to the bottom of the tube. At the end of the centrifugation, the media were transferred from the falcon tube to the waste container and $3 \mathrm{mM}$ of medium was added onto the cell sediment. Cells collected in the bottom of the tube were suspended with a sterile pipette. The Cedex Hires Analyzer (Roche) device was used for cell counting. Dead cells were labelled with trypan blue solution. The obtained data were used to determine the number of cells to be added to the wells of the E-Plate 96 plate.

\subsubsection{Antiproliferatif Activity Tests}

Antiproliferative activity tests were performed using real-time cell analyzer (xCelligence RTCA SP, ACEABIO, Inc.). RTCA SP is based on the principle of monitoring cell biology by microelectronic technique. The RTCA SP (Single Plate) unit consists of RTCA control unit, RTCA analyzer, RTCA SP station and EPlate 96 components. One of the most important parts of the RTCA SP is the plate with 96 wells (E-Plate 96). At the bottom of the plate is a microelectronic cell sensor array suitable for cells adhesion. Biological alterations in the cells cause resistance changes on the RTCA SP sensors.

This resistance change is measured automatically and converted to digital signals so that the reader can read and analyze it. Cell viability, cell number, cell morphology and the attraction of molecules to each 
other (adhesion) affect electrode resistance. If there is a cell on the electrode, the cell attached to the electrode surface behaves as insulators and cause an increase in the resistance of the medium. This means that the more cells there are on the electrode, the greater the change in resistance on the electrode. The cell index ( $\mathrm{Cl}$, Cell Index) parameter is derived from the relative position of the cell state. The $\mathrm{Cl}$ value is approximately zero when cells are not present or not well attached to the electrodes. If more cells are attached on the electrode in the same physiological conditions, the $\mathrm{Cl}$ value is higher [36]. $\mathrm{Cl}$ (Cell Index) is a unitless value. As the value of $\mathrm{Cl}$ increases, it is understood that the cells hold onto the surface, they develop, the morphology does not show any change, and they divide and multiply. In other words, an increase in the $\mathrm{Cl}$ value indicates that the cells are not deformed, that the cells do not enter the stress, that the conditions for the cells are appropriate and that there are no problems about proliferation.

Cells are added to the wells of E-Plate 96 , which has previously been pre-filled with a certain amount of medium for each well. Within a short time, the cells touch and adhere to the surface of the sensorelectrode located at the bottom of the wells. The electronic properties of the sensor surfaces are monitored via the station located within a $\mathrm{CO}_{2}$ incubator. Simultaneous quantitative information about the biological state of the cell such as cell viability, morphology and cytotoxicity is determined by the RTCA software in the RTCA analyzer and the control unit.

Antiproliferative activity studies were carried out according to the method used by Abay [37]. Samples were dissolved in sterile DMSO (as $20 \mathrm{mg} / \mathrm{mL}$ ) and diluted in sterile tubes with DMEM (1:20). $50 \mu \mathrm{l}$ culture medium (DMEM) was added in each well of E-plate 96 . The plate was incubated in sterile cabinet for 15 minutes and then in the $\mathrm{CO}_{2}$ incubator for $15 \mathrm{~min}$ (for the thermal balance). Subsequently, the plate was placed in the RTCA station and a background reading was performed. This reading lasted 1 minute and the status of E-plate 96 was evaluated. The counting of the cells to be added to the wells and the preparation of the suspension was carried out using with the cell counting device. $100 \mu$ of this cell suspension was added to each well of the plate $(25.000$ cells/well) except last three wells. These wells were left without cells to check if there would be a $\mathrm{Cl}$ change or not due to DMEM. The plate was reinserted in the RTCA station and the second phase ( $80 \mathrm{~min}$.) was started. During this time, the cells were allowed to adhere to the bottom of the well and enter the growth process. At the end of this period, the e-plate was taken back to the sterile cabinet and the culture medium solution containing the molecule samples was added to the wells at different concentrations. The samples solutions were added to the wells at three different concentrations as 100,50 , and $10 \mu \mathrm{g} / \mathrm{mL}$. The final volume of each well was adjusted to $200 \mu \mathrm{l}$ with DMEM. Each dose was studied in triplicate. Then, the plate was placed on the RTCA for the last time. The life/death states of the cells were recorded for 48 hours. The standard deviation of triplicates of wells were analyzed by the RTCA Software.

\section{Results}

\subsection{Chemistry}


The primary target of this study was to develop antimicrobial hybrid substances covering various pharmacophore structures. Reactions of last and intermediate compounds were achieved as pictorial in Scheme 1, Scheme 2, Scheme 3, and Scheme 4. The synthesis was carried out by utilizing microwaveassisted and conventional methods. The finishing of the synthesis was observed via the thin-layer chromatography (TLC) process. All product structures were based on the foundation of spectral and physicochemical data.

\subsection{Antimicrobial Activity}

All newly products were tested for their antimicrobial properties utilizing the minimal inhibition concentration method (MIC) and the outcomes for active molecules are illustrated in Table 1. The antimicrobial activities of compounds (2-9) were tested against 4 bacteria and 3 yeasts.

Table 1. Antimicrobial activity of the compounds $(\mu \mathrm{g} / \mathrm{mL})$ 


\begin{tabular}{|c|c|c|c|c|c|c|c|c|c|}
\hline \multirow{2}{*}{$\begin{array}{l}\text { Comp. } \\
\text { No. }\end{array}$} & \multicolumn{9}{|c|}{ Minimal Inhibition Concentration Values $(\mu \mathrm{g} / \mathrm{mL})$} \\
\hline & Ec & $Y p$ & $\mathrm{~Pa}$ & $\mathrm{Sa}$ & Ef & $\mathrm{Bc}$ & Ms & $\mathrm{Ca}$ & $\mathrm{Sc}$ \\
\hline 3 & - & - & 500 & - & - & - & - & 500 & - \\
\hline 5 & - & - & - & - & - & - & - & 125 & - \\
\hline $6 c$ & - & - & - & - & 250 & - & - & - & - \\
\hline $7 a$ & 125 & - & - & - & - & - & - & - & - \\
\hline $7 b$ & - & - & - & - & - & - & 62.5 & - & - \\
\hline $7 c$ & - & - & - & - & - & - & - & 125 & 250 \\
\hline $8 b$ & 62.5 & - & - & 125 & - & 125 & 1.95 & - & - \\
\hline $9 a$ & 0.24 & - & 500 & - & - & - & - & - & - \\
\hline $9 b$ & 250 & - & - & - & - & - & - & - & - \\
\hline $10 a$ & - & - & - & - & 250 & - & - & - & - \\
\hline $10 b$ & - & - & - & - & - & - & - & 625 & - \\
\hline $10 d$ & - & - & - & - & - & - & 125 & - & - \\
\hline $10 \mathrm{e}$ & - & - & - & - & - & - & - & 625 & - \\
\hline $11 a$ & $<1$ & 1.9 & 3.9 & - & 3.9 & 7.8 & 3.9 & - & - \\
\hline $11 \mathrm{~b}$ & 1.9 & 62.5 & 15.6 & - & 15.6 & 31.25 & 31.25 & - & - \\
\hline $11 \mathrm{c}$ & - & 62.5 & - & - & - & - & 62.5 & 312.5 & 312.5 \\
\hline $11 d$ & 62.5 & - & 625 & - & - & - & - & 312.5 & 312.5 \\
\hline $11 \mathrm{e}$ & 15.6 & - & $>1000$ & - & - & - & - & 312.5 & 156 \\
\hline 12 & - & - & 500 & - & - & 500 & - & 250 & - \\
\hline $13 a$ & $<1$ & 7.8 & - & 156 & 19 & 39 & 3.9 & 312.5 & 78 \\
\hline $13 b$ & 31.25 & $<1$ & - & 9.7 & 9.7 & 9.7 & $<1$ & 312.5 & 312.5 \\
\hline $14 a$ & $<1$ & $<1$ & $<1$ & - & $<1$ & 1.9 & $<1$ & - & - \\
\hline $14 b$ & $<1$ & $<1$ & $<1$ & - & $<1$ & $<1$ & $<1$ & - & - \\
\hline $14 c$ & $<1$ & $<1$ & $<1$ & - & $<1$ & 1.9 & $<1$ & - & - \\
\hline $14 d$ & $<1$ & $<1$ & 1.9 & - & - & 1.9 & 1.9 & - & - \\
\hline $14 \mathrm{e}$ & $<1$ & $<1$ & 3.9 & - & $<1$ & 1.9 & $<1$ & - & - \\
\hline
\end{tabular}




\begin{tabular}{|llllllllll|} 
Amp. & 10 & 18 & $>128$ & 35 & 10 & 15 & & & \\
\hline Strep. & & & & & & & 4 & & \\
\hline Flu. & & & & & & & & $<8$ & $<8$ \\
& & & & & & & & & \\
\hline
\end{tabular}

Ec: E. coli ATCC 35218, Yp: Y. pseudotuberculosis ATCC 911, Pa: P. aeruginosa ATCC 10145, Sa: S. aureus ATCC 25923, Ef: E. faecalis ATCC 29212, Bc: B. cereus 709 Roma, Ms: M. smegmatis ATCC607, Ca: C. albicans ATCC 60193, S. cerevisiae RSKK 251, Amp.: Ampicillin, Strep.: Streptomycin, Flu.: Fluconazole, $(-)$ : no activity of test concentrations

Among the synthesized compounds, compounds 3-9 displayed modarete activity in relation to test microorganisms while showing better activities on the M. smegmatis and E. coli. That is compounds $7 \mathbf{b}$ and $\mathbf{8 b}$ have more effective for $M$. smegmatis than the other molecules with the MIC values of 62.5 and $1.95 \mu \mathrm{g} / \mathrm{mL}$ respectively. $M$. smegmatis is one of an acid-fast, aerobic-rapidly growing bacterial species in the genus Mycobacterium and constitutes one of a characteristic tuberculosis agent causing the death and morbidity. On the other hand, compound $9 \mathrm{a}$, an intermediate, showed excellent activity on $E$. coli, a gram-negative bacterium, at $0.24 \mu \mathrm{g} / \mathrm{mL}$ MIC value. Namely 9 a exhibited much better activity against to E. coli than ampicillin used as standard drug. E. coli which is rod-shaped, gram-negative enteric bacteria which lives in the enteric systems of humans and animals and are found in aqua and fecal substance.

Compounds $10 \mathrm{a}-\mathrm{e}$ which are conazole derivatives exhibited slight activity on the gram-positive and gramnegative bacteries and yeasts. But compound $10 \mathrm{c}$ no antimicrobial effects were found towards to the test microorganisms.

Mannich bases 11 ae displayed good antibacterial activity to test microorganisms. However these compounds presented not good antimicrobial effect against the yeast strains $C$. albicans and $S$. cerevisiae. Only compound $11 \mathrm{e}$, containing phenyl piperazine, showed moderate activity MIC value of 156 $\mu \mathrm{g} / \mathrm{mL}$ on $S$. cerevisiae.

Oxadiazole compound, 12 , obtained as a result of ring closure, has low activity on all the microrganisms. But, mannich bases $13 \mathbf{a}, \mathbf{b}$ obtained from the reaction of the oxadiazole compound with the quinolone showed strong activities. Especially compound $13 \mathbf{a}$ exhibited the best activity among all compounds synthesized against yeast strains with $78 \mu \mathrm{g} / \mathrm{mL}$ MIC value on $S$. cerevisiae. That is 13 a has a good antifungal activity.

Among the synthesized compounds, 11 a-e mannich bases demonstrated the best and excellent activity against gram-positive and gram-negative bacteria with MIC values betweena $<1$ and $31.25 \mu \mathrm{g} / \mathrm{mL}$, while showing no activity against yeast strains. At the same time, these compounds showed very good antitubercular activity against $M$. smegmatis compared with Streptomycin standard drug. 
No antimicrobial effects were found against to the test microorganisms in the studied concentration ranges of the $4,6 a, b, 8 a$ and $10 \mathrm{c}$ compounds. Therefore, these results are not included in the table.

\subsection{Anticancer Activity}

The antiproliferative activity potentials of the molecules were examined by Abay's [37] method against HeLa cells. Three different doses of the samples showed different antiproliferative activity against the cells (Fig. 1 and Fig. 2). High Cell Index (Cl) values (red line) were obtained from the wells without the molecules samples. Lower $\mathrm{Cl}$ values were obtained from the wells in which the molecules were added. Only the medium (DMEM) was added to the final three wells. These three wells were used to obtain a baseline. No impedance change was observed in these wells containing only DMEM. Therefore, the Cell Index $(\mathrm{Cl})$ values of these three wells did not increase at all and straight continued to the end of the experiment (green line) similarly.

$\mathrm{Cl}$ values obtained from only DMEM and cell-added wells were shown as the red line. The HeLa cells that continue to develop without encountering any obstacles have caused the $\mathrm{Cl}$ to rise rapidly. This situation shows that the cells are attached to gold-plated microelectrodes in the well ground during the experiment and there is no proliferative negativity. The more the cells attached to the electrodes on the plate floor, the greater the impedance change. This results in an increase in $\mathrm{Cl}$ value. On the contrary, the decrease in $\mathrm{Cl}$ value means that the proliferation of the cells is suppressed or inhibited.

The hard $\mathrm{Cl}$ decline seen at the $2 \mathrm{nd}$ hour is due to the change in the temperature of the e-Plate 96 taken from the RTCA station in the incubator $\left(95 \% \mathrm{CO}_{2}, 37^{\circ} \mathrm{C}\right)$ to the sterile cabinet and the sample addition. After the addition of the molecule samples, the E-Plate 96 inserted into the station in the $\mathrm{CO}_{2}$ incubator again. The cells in the wells with no added samples were rapidly increased the $\mathrm{Cl}$ values (red line). However, the proliferation of the cells in the wells the samples were added was strongly suppressed by the samples' molecules. This situation resulted in low $\mathrm{Cl}$ values.

80 minutes after the addition of the cells to the wells, the e-plate was removed from the station and taken to the sterile cabinet. The samples were added to the wells three different concentrations $(100,50$ and 10 $\mu \mathrm{gL}^{-1}$ ). Each doses of the samples were evaluated in triplicate. The antiproliferative effect of the molecules against HeLa cells was monitored in real time every 10 minutes and followed for 48 hours. The mean $\mathrm{Cl}$ values from the wells were calculated automatically with the xCelligence RTCA SP software and the standard deviations are shown as vertical bars.

Dose effect investigations are performed by considering terms such as a dose dependent effect, dosedependent reverse effect, hormesis and inverse hormesis [38-40]. Antiproliferative effects of these molecules against HeLa cells are shown in Fig. 1 and Fig. 2. Cl values obtained from wells with no cells increased rapidly (red line). Different doses of DMSO were added to the wells with HeLa cells and no DMSO-induced antiproliferative activity was observed (Fig. 3). The effect of all doses of 5FU used as a positive control was strong, and $\mathrm{Cl}$ values were very close to each other. None of the lower doses of the molecules didn't showed antiproliferative effect $\left(10 \mu \mathrm{g} \mathrm{mL}^{-1}\right.$, turquoise line). All of the low doses of the 
molecules produced the same $\mathrm{Cl}$ as the control group. Only the low dose of compound 8a partially dissociated from the control group towards the end of the experiment (after 35 hours). However, this situation cannot be considered as a net antiproliferative effect. On the 5FU graph (Fig. 4) used as a positive control, it is seen that the low dose (turquoise line) has a clearly different $\mathrm{Cl}$ value from the red colored $\mathrm{Cl}$ values (negative control).

Middle doses of $\mathbf{8 a}$ and $\mathbf{9 b}$, molecules ( $50 \mathrm{\mu g} \mathrm{mL}^{-1}$, pink line) showed strong antiproliferative activity. They maintained their antiproliferative effects throughout the experiment. High doses of molecules have also been found to have high antiproliferative activities. High doses of the $9 \mathrm{~b}$ molecule $(100 \mu \mathrm{g} \mathrm{mL}-1$, dark blue line) showed very strong antiproliferative activity. High doses of the $\mathbf{8 a}$ molecule also showed a strong antiproliferative effect $\left(100 \mu \mathrm{g} \mathrm{mL}^{-1}\right.$, dark blue line) on HeLa cells. The effects of high doses of the $9 \mathrm{~b}$ molecules were so potent that the resulting $\mathrm{Cl}$ values were the same as the $0 \mathrm{Cl}$ values (green line) obtained from wells with no added cells. This strong effect continued throughout the experiment.

The dose effect differences of these molecules were most clearly seen in $\mathbf{8 a}$ and $\mathbf{9 b}$. These effects of molecules with strong antiproliferative effects are due to their structural forms. The antiproliferative activity potentials of the molecules that didn't show any effect or have a weak effect against to HeLa cells should be examined against other cancer cell lines and their effects should be investigated.

\section{Discussion}

Schiff base (3) was obtained from the reaction of 3-alkyl-4-amino-4,5-dihydro-1H-1,2,4-triazol-5-one (2) compound with cinnamaldehyde, which was obtained from the reaction of ester ethoxycarbonylhydrazone (1) and hydrazine hydrate [34]. The object was to combine the 1,2,4-triazole nucleus to the cinnamaldehyde since it is known that more efficacious antimicrobial substances can be discovered by adding two biologically effective components together into a single molecular structure $[41,42]$. Thereafter product (3) was transformed to the corresponding hydrazide derivative (5) by the creation of ester (4). With introduction of hydrazide function in the molecule, two signals appeared at 4.23 anda0.88 ppm as $\mathrm{D}_{2}$ Oaexchangeable singlets at the ${ }^{1} \mathrm{H}$ NMRadata of molecule (5). With introduction of hydrazide group in the compound, two signals emerged at 5.25 and $9.17 \mathrm{ppm}$ as $\mathrm{D}_{2} \mathrm{O}$ exchangeable singlets at the ${ }^{1} \mathrm{H}$ NMR data of molecule.

Molecules (6a-c) were performed by the treatment of molecule (5) with phenyl- (for 6a), or benzyl isothiocyanate (for $\mathbf{6 b}$ ), or phenyl isocyanate (for $6 \mathrm{c}$ ) in dichlomethanolic solution in good yields and molecule constructions were confirmed via FT IR, ${ }^{1} \mathrm{H}$ NMR, ${ }^{13} \mathrm{C}$ NMR, mass data.

The intramolecular cyclization of products $(6 a-c)$ at basic media afforded in the transformation of carbox(thio)amide function alter to 5-oxo(mercapto)-1,2,4-triazole derivatives and so molecules 7a-c were synthesized. These compounds were characterized by the presence of a signal at 13.70 and $14.02 \mathrm{ppm}$ in the ${ }^{1} \mathrm{H}$ NMR data as a $\mathrm{D}_{2} \mathrm{O}$ exchangeable singlet confirming the existence of a $-\mathrm{SH}$ function $(7 \mathrm{a}, \mathrm{b})$ and 
$10.77 \mathrm{ppm}-\mathrm{NH}$ function (for 7c). The stretching band derived from this groups appeared at 2929 and $2932 \mathrm{~cm}^{-1}$, and C $=0$ (for 7c) observed at $1626 \mathrm{~cm}^{-1}$ at the FT-IR data of these molecules (Scheme 1).

Alkylation of products 3, viaa2-bromo-1-(4-chlorophenyl)-ethanone or 2-chloro-1-(2,4-dichlorophenyl)ethanone in ethanol performed compound $\mathbf{8 a}, \mathbf{b}$. These reactions, which took place in 1440 minute in the conventional method, took place in 6 minute in the microwave irradiated method. Studies related to microwave synthesis which support organic synthesis in a shorter time and higher efficiency are available in the literature [43-45]. $\mathrm{NH}$ proton attached to the triazole group disappeared for compound $\mathbf{3}$ at thea ${ }^{1} \mathrm{H}$ NMR spectra. New aromatic peaks were resonated in the region $7.27-7.67 \mathrm{ppm}$. In ${ }^{13} \mathrm{C}$ NMR datas of molecules, the carbon atom $(C=0)$ were observed between 192.56 and $194.43 \mathrm{ppm}$ for the newly added carbonyl group.

Compounds $\mathbf{9 a , b}$ was obtained with the reduction of the carbonyl structure of products $\mathbf{8 a}, \mathbf{b}$ with sodium borohydride utilizing both classical heating and MWairradiation. When we compare the traditional and microwave method MW irradiation reduced the reaction time froma960 min. to $8 \mathrm{amin}$. and increased the yields. Looking at compound number $9 a, b$, the carbonyl group peak has evanesced at the ${ }^{1} \mathrm{H}$ NMR and ${ }^{13} \mathrm{C}$ NMR datas and $\mathrm{OH}$ peak added between 5.41 and $6.44 \mathrm{ppm}$ in the ${ }^{1} \mathrm{H}$ NMR. The spreading band obtained this group $(\mathrm{OH})$ appeared between 3374 and $3251 \mathrm{~cm}^{-1}$, in the FT-IR data of molecules.

Reactions of molecules $10 \mathrm{a} e$, were afforded reaction of molecule $9 \mathrm{a}, \mathrm{b}$ and benzyl chlorides, such as 2,4dichloro-, 2,6-dichloro- and 4-chlorobenzyl,chlorides in a ambiance with of $\mathrm{NaH}$ via MW sythesis method at $80^{\circ} \mathrm{C}$ and $100 \mathrm{~W}$ for $5 \mathrm{~min}$. In both FT-IR and ${ }^{1} \mathrm{H}$ NMR datas of the molecules, the peaks due to the $-\mathrm{OH}$ group have disappeared. Another peaks approving molecule structures were displayed at the concerned chemical ranges in the ${ }^{1} \mathrm{H}$ NMR and ${ }^{13} \mathrm{C}$ NMR spectra. Moreover, $[\mathrm{M}+1]$ ion signals were appeared at the concerned $\mathrm{m} / \mathrm{z}$ ranges auxiliaring the offered structures of molecules $10 \mathrm{a}-\mathbf{e}$ (Scheme 2 ).

Oxadiazole compound (12) was obtained as a result of ring closure reaction of hydrazide compound (5) with $\mathrm{CS}_{2}$ in basic media. The $\mathrm{NH}$ and $\mathrm{NH}_{2}$ peaks resulting from the hydrazide compound disappeared in both the FT-IR and ${ }^{1} \mathrm{H}$ NMR data of the oxadiazol derivative compound. Instead of those peaks, SH peaks were added at $14.66 \mathrm{ppm}$ in ${ }^{1} \mathrm{H}$ NMR and $2749 \mathrm{~cm}^{-1}$ in the FT-IR. In addition synthesized molecules confirmed ${ }^{13} \mathrm{C}$ NMR and Mass spectral data and elemental analysis results consistent with the assigned sutructures (Scheme 3).

Mannich reaction is a three-component condensation reaction involving active hydrogen containing compound, formaldehyde and a secondary amine. The amino alkylation of aromatic substrates by Mannich reaction is of considerable importance for the synthesis and modification of biologically active compounds [46]. Mannich bases found numerous practical applications in the field of medicinal chemistry, it could be responsible for enhancing physicochemical properties [47]. Mannich bases linked 1,2,4-triazole derivatives as containing a significant biological activity that has been reported in the literature $[48,49]$. Furthermore, several Mannich bases of triazole derivatives including piperazine, thiomorpholine, or morpholine moiety were synthesized as antimicrobial agents in our laboratory [50,51]. 
Fluoroquinolones are known as the most broadly utilized synthetic antimicrobial substances; privileged with wide spectrum antibacterial property, relatively low occurrence of toxic and adverse effects along with an perfect safety profile [47].

Considering these facts in this research, the aminoalkylation of structures $3,7 a-c$, and 12 with different amines, such as norfloxacin (for 11a, 13a, 14a, 14b), ciprofloxacin (for 11b, 13b, 14c, 14d, 14e), morpholine (for 11c), thiomorpholine (11d), and 4-phenylpiperazine (for 11e) in an ambiance with formaldehyde was performed using the MW-assisted Mannich synthesis reactions (Scheme 2, Scheme 3 and Scheme 4). No signal symbolizing the presence of the $\mathrm{NH}$ band exists on the ${ }^{1} \mathrm{HNMR}$ and FT-IR spectra of products (11a-e, 13a,b, 14a-e) and in the ${ }^{1} \mathrm{H}$ NMR and ${ }^{13} \mathrm{C}$ NMR spectra of molecules extra signals originated from amine moieties were observed at the concerned chemical ranges. These molecules displayed mass spectral datum records reasonable with their constructions.

The use of microwave (MW) irradiation method consequences in very influential and clean results with notable developments compared to classical processes. The process via MW irradiation ensured the more helpful road with developed synthesis yields and shorter synthesis times [54]. Green Chemistry would like the high yield of synthetic processes, the use of less toxic solvents, and the decrease in phases of synthetic schemes [53].

\section{Conclusions}

This study statements the synthesis of novel compounds having various bioactive units via microwave irridation and conventional techniques. Microwave method provided more efficient way the synthesis of desired compounds. Also, antibacterial, and antiproliferative activity of the synthesized molecules were determined. Among the synthesized compounds, the best antimicrobial activities were found to show compounds $14 \mathrm{a}-\mathrm{e}$ which are Mannich bases. Especially these compounds showed very good antitubercular activity against $M$. smegmatis compared with Streptomycin standard drug. And also they exhibited better activity against to $E$. colithan ampicillin used as standard drug. Middle and high doses of compound $\mathbf{8 a}$ and $\mathbf{9 b}$ were found to have strong anticancer activity on the HeLa cervical cancer cells.

\section{Declarations}

\section{Acknowledgement}

The support provided by Scientific and Technological Research Council of Turkey (TUBITAK, Project no:a113Z181).

\section{Conflict of Interest}

The authors declare no conflict of interest.

\section{References}


1. Moshawih S, Mydin RBS, Kalakotla S, Jarrar QB (2019) Potential application of resveratrol in nanocarriers against cancer: Overview and future trends. J Drug Deliv Sci Technol 53:101187

2. Stucci S, Palmirotta R. Passarelli A, Silvestris E, Argentiero A, Lanotte L, Silvestris F (2017) Immunerelated adverse events during anticancer immunotherapy: Pathogenesis and management. Oncol Lett 14(5):5671-5680

3. Gorle S, Gangu KK, Maddila S, Jonnalagadda SB (2020) Synthesis and anticancer activity of novel pyrazolo [4', 3': 5, 6] pyrano [2, 3-d] pyrimidin-5 (2H)-one derivatives. Chemical Data Collections 28:100471

4. Bray F, Møller B (2006) Predicting the future burden of cancer. Nat Rev Cancer 6(1):63-74

5. Fouad MM, El-Bendary ER, Shehata IA, El-Kerdawy MM (2018) Synthesis and in vitro antitumor evaluation of some new thiophenes and thieno [2, 3-d] pyrimidine derivatives. Bioorg Chem 81:587598

6. Wellington KW (2015) Understanding cancer and the anticancer activities of naphthoquinones-a review. RSC Adv 5(26):20309-20338

7. Jamalian A, Shafiee A, Hemmateenejad B, Khoshneviszadeh M, Miri R. Madadkar-Sobhani A, Bathaie S, Moosavi-Movahedi AA (2011) Novel imidazolyl derivatives of 1, 8-acridinedione as potential DNAintercalating agents. J Iran Chem Soc 8(4):1098-1112

8. Levin-Reisman I, Ronin I, Gefen O, Braniss I, Shoresh N, Balaban NQ (2017) Antibiotic tolerance facilitates the evolution of resistance. Science 355:826-830

9. Sprenger M, Fukuda K (2016) New mechanisms, new worries. Science 351:1263-1264

10. Brown ED, Wright GD (2016) Antibacterial drug discovery in the resistance era. Nature 529:336-343

11. Silver SL (2011) Challenges of antibacterial discovery. Clin Microbiol Rev 24:71-109

12. Cho S, Kim SH, Shin D (2019) Recent applications of hydantoin and thiohydantoin in medicinal chemistry. Eur J Med Chem 164:517-545

13. Meunier B (2008) Hybrid molecules with a dual mode of action: dream or reality? Acc Chem Res 41(1):69-77

14. Mishra S, Singh P (2016) Hybrid molecules: The privileged scaffolds for various pharmaceuticals. Eur J Med Chem 124:500-536

15. Shneine JK, Alaraji YH (2016) Chemistry of 1,2,4-triazole: a review article. Int J Recent Sci Res 5:1411-1423

16. Namratha B, Gaonkar SL (2014) 1,2,4-Triazoles: synthetic strategies and pharmacological profiles. Int J Pharm Pharmaceut Sci 6:73-80

17. Mermer A, Demirbas N, Demirbas A, Colak N, Ayaz FA, Alagumuthu M, Arumugam S (2018) Synthesis, biological activity and structure activity relationship studies of novel conazole analogues via conventional, microwave and ultrasound mediated techniques. Bioorg Chem 81:55-70

18. Almasirad A, Tabatabai SA, Faizi M, Kebriaeezadeh A, Mehrabi N, Dalvandi A, Shafiee A (2004) Synthesis and anticonvulsant activity of new 2-substituted-5-[2-(2-fluoro phenoxy)phenyl]-1,3,4- 
oxadiazoles and 1,2,4-triazoles. Bioorg Med Chem Lett 14:6057-6059

19. Hanif M, Saleem M, Hussain MT, Rama NH, Zaib S, Aslam MAM, Jones PG, Iqbal J (2012) Synthesis, urease inhibition, antioxidant and antibacterial studies of some 4-amino-5-aryl-3H-1,2,4-triazole-3thiones and their 3,6-disubstituted 1,2,4-triazolo[3,4-b]1,3,4-thiadiazole derivatives. J Braz Chem Soc 23:854-860

20. Dündar Y, Çakır B, Küpeli E, Sahin MF, Noyanalpan N (2007) Synthesis of some new 1acylthiosemicarbazides and 1,2,4-triazol-5-thiones, and their analgesic and anti-inflammatory activities. Turk J Chem 31:301-313

21. Sitaram D, Celik G, Khloya P, Vullo D, Supuran CT, Sharma PK (2014) Benzenesulfonamide bearing 1,2,4-triazole scaffolds as potent inhibitors of tumor associated carbonic anhydrase isoforms hCA IX and hCA XII. Bioorg Med Chem 22:1873-1882

22. Küçükgüzel I, Tatar E, Küçükgüzel SG, Rollas S, De Clercq E (2008) Synthesis of some novel thiourea derivatives obtained from 5-[(4-aminophenoxy)methyl]-4-alkyl/aryl-2,4-dihydro-3H-1,2,4-triazole-3thiones and evaluation as antiviral/anti-HIV and anti-tuberculosis agents. Eur J Med Chem 43:381392

23. Saadeh HA, Mosleh IM, Al-Bakri AG, Mubarak MS (2010) Synthesis and antimicrobial activity of new 1,2,4-triazole-3-thiol metronidazole derivatives. Monatsh Chem 141:471-478

24. Turan-Zitouni G, Kaplancikli ZA, Kiliç FS, Erol K (2002) The synthesis of some triazolylphenothiazine derivatives and their antidepressant and anxiolytic activities. Boll Chim Farm 141:192-196

25. Sissi C, Palumbo M (2003) The quinolone family: from antibacterial to anticancer agents. Curr Med Chem Anticancer agents 3:439-450

26. Afzal O, Kumar S, Haider MR, Ali MR, Kumar R, Jaggi M, Bawa S (2015) A review on atnicancer potential of bioactive heterocycle quinolone. Eur J Med Chem 97:871-910

27. Zhang GF, Liu X, Zhang S, Pan B, Liu ML (2018) Ciprofloxacin derivatives and their antibacterial activities. Eur J Med Chem 146:599-612

28. Zhang GF, Zhang S, Pan B, Liu X, Feng LS (2018) 4-Quinolone derivatives and their activities against Gram positive pathogens. Eur J Med Chem 143:710-723

29. Kjærstad MB, Taxvig C, Nellemann C, Vinggaard AM, Andersen HR (2010) Endocrine disrupting effects in vitro of conazole antifungals used as pesticides and pharmaceuticals. Reprod Toxicol 30(4):573-582

30. Fiorot RG, Allochio Filho JF, Pereira TM, Lacerda V Jr, dos Santos RB, Romão W, Greco SJ (2014) A simple and convenient method for synthesis of new aminonaphthoquinones derived from lawsone by catalytic multicomponent Mannich reaction. Tetrahedron Lett 55(31):4373-4377

31. Mansoor SS, Aswin K, Logaiya K, Sudhan SPN (2015) An efficient synthesis of $\beta$-amino ketone compounds through one-pot three-component Mannich-type reactions using bismuth nitrate as catalyst. J Saudi Chem Soc 19:379-386

32. Sivakumar KK, Rajasekaran A, Senthilkumar P, Wattamwar PP (2014) Conventional and microwave assisted synthesis of pyrazolone Mannich bases possessing anti-inflammatory, analgesic, 
ulcerogenic effect and antimicrobial properties. Bioorg Med Chem Lett 24:2940-2944

33. War JA, Srivastava SK, Srivastava SD (2017) Design, synthesis and DNA-binding study of some novel morpholine linked thiazolidinone derivatives. Spectrochim Acta Part A: Mol Biomol Spectrosc 173:270-278

34. Demirbas N, Ugurluoglu R, Demirbas A (2002) Synthesis of 3-Alkyl(Aryl)-4-alkylidenamino-4,5dihydro-1H-1,2,4-triazol-5-ones and 3-Alkyl-4-alkylamino-4,5-dihydro-1H-1,2,4-triazol-5-ones as Antitumor Agents. Bioorg Med Chem 10:3717-3723

35. Willanova PA. National Committee for Clinical Laboratory Standard, NCCLS Document M7-A3, 13, 1993

36. Ke N, Wang X, Xu X, Abassi YA (2011) The xCELLigence system for real-time and label-free monitoring of cell viability Methods. Mol Biol 740:33-43

37. Abay G, Altun M, Koldas S, Tufekci AR, Demirtas I (2015) Determination of antiproliferative activities of volatile contents and HPLC profiles of Dicranum scoparium (Dicranaceae, Bryophyta). Comb Chem High Throughput Screen 18:453-463

38. Cordell GA (2000) Biodiversity and drug discovery-A symbiotic relationship. Phytochemistry 55:463480

39. Naman CB, Leber CA, Gerwick WH (2017) Modern Natural Products Drug Discovery and Its Relevance to Biodiversity Conservation. In: Kurtböke I (ed) Microbial Resources: From Functional Existence in Nature to Applications. Academic Press, pp 103-120

40. Shen B (2015) A New Golden Age of Natural Products Drug Discovery. Cell 163:1297-1300

41. Mishra S, Singh P (2016) Hybrid molecules: The privileged scaffolds for various pharmaceuticals. Eur J Med Chem 124:500-536

42. Tietze LF, Bell HP, Chandrasekhar S (2003) Natural Product Hybrids as New Leads for Drug Discovery. Angew Chem Int Ed 42:3996-4028

43. Yi X, Zhang Z, Huang H, Baell JB, Yu Y, Huang F (2019) Microwave-Assisted Synthesis of a Diazoesters. Chin J Org Chem 39(2):544

44. Dandia A, Gupta SL, Sharma R, Saini P, Parewa V (2021) Microwave-assisted catalyst-free organic synthesis. In: Green Sustainable Process for Chemical and Environmental Engineering and Science. Elsevier, pp 539-622

45. Saini N, Sharma A, Thakur VK, Makatsoris C, Dandia A, Bhagat M, Sharma PC (2020) Microwave assisted green synthesis of thiazolidin-4-one derivatives: A perspective on potent antiviral and antimicrobial activities. Current Research in Green Sustainable Chemistry 3:100021

46. Ashok M, Holla BS, Poojary B (2007) Convenient one pot synthesis and antimicrobial evaluation of some new Mannich bases carrying 4-methylthiobenzyl moiety. Eur J Med Chem 42(8):1095-1101

47. Abdel-Rahman IM, Mustafa M, Mohamed SA, Yahia R, Abdel-Aziz M, Abuo-Rahma GEDA, Hayallah AM (2021) Novel Mannich Bases of Ciprofloxacin with Improved Physicochemical Properties, Antibacterial, Anticancer Activities and Caspase-3 Mediated Apoptosis. Bioorg Chem 107:104629 
48. Plech T, Wujec M, Kosikowska U, Malm A, Rajtar B, Polz Dacewicz M (2013) Synthesis and in vitro activity of 1, 2, 4-triazole-ciprofloxacin hybrids against drug-susceptible and drug-resistant bacteria. Eur J Med Chem 60:128-134

49. Wang BL, Liu XH, Zhang XL, Zhang JF, Song HB, Li ZM (2011) Synthesis, structure and biological activity of novel 1, 2, 4-triazole mannich bases containing a substituted benzylpiperazine moiety. Chem Biol Drug Des 78:42-49

50. Ozyanık M, Demirci S, Bektas H, Demirbas N, Demirbas A, Alpay-Karaoglu S (2012) Preparation and antimicrobial activity evaluation of some quinoline derivatives containing an azole nucleus. Turk $\mathrm{J}$ Chem 36:233-246

51. Fandaklı S, Basoglu S, Bektas H, Yolal M, Demirbas A, Alpay-Karaoglu S, Reduction (2012) Mannich reaction, and antimicrobial activity evaluation of some new 1,2,4-triazol-3-one derivatives. Turk J Chem 36:567-582

52. Loupy A (2004) Solvent-free microwave organic synthesis as an efficient procedure for green chemistry. Cr Chimie 7(2):103-112

53. Grewal AS, Kumar K, Redhu S, Bhardwaj S (2013) Microwave assisted synthesis: a green chemistry approach. Int Res J Pharm App Sci 3(5):278-285

\section{Schemes}

Schemes 1-4 are in the Supplemental Files section.

\section{Figures}

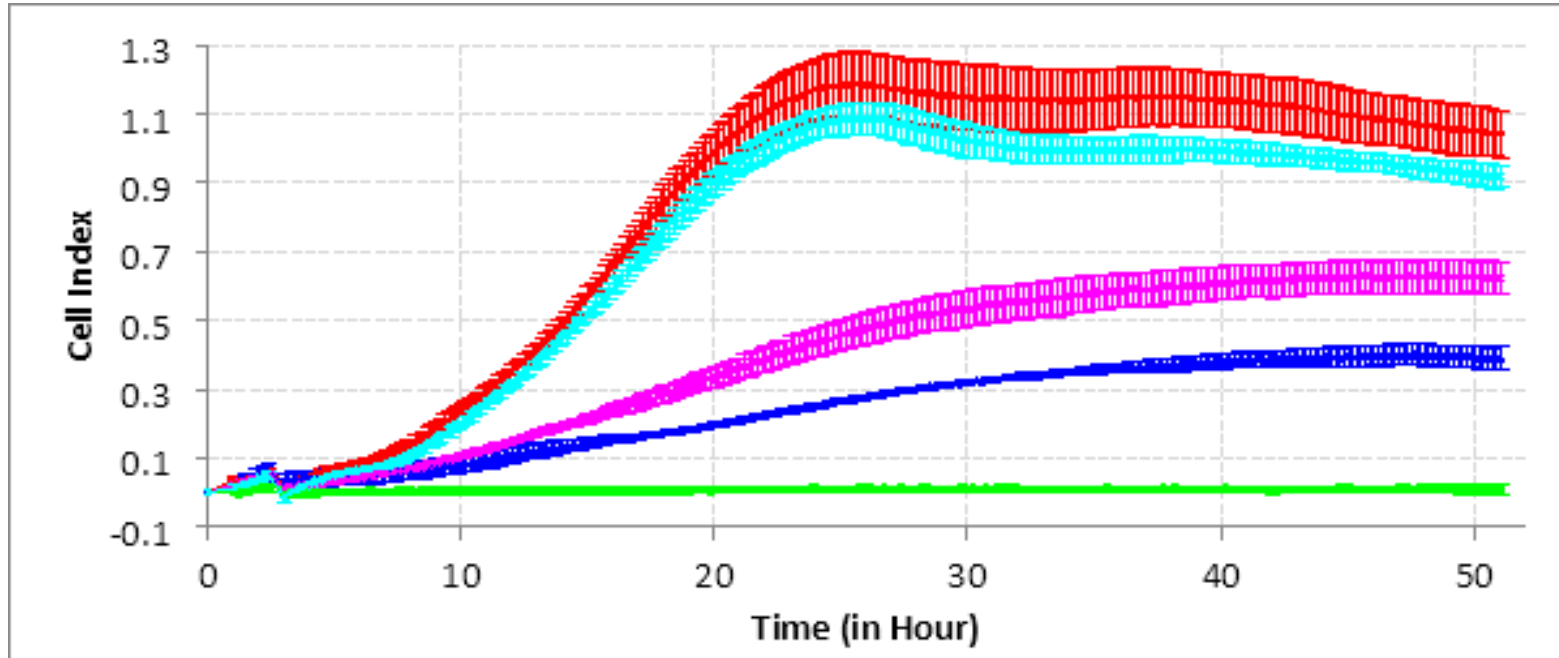

\section{Figure 1}

Time-Cl (Cell Index) plot showing the antiproliferative activity test results of Compound 8a against HeLa cell line. (The concentration unit is $\mu \mathrm{g} / \mathrm{mL}$ ). 


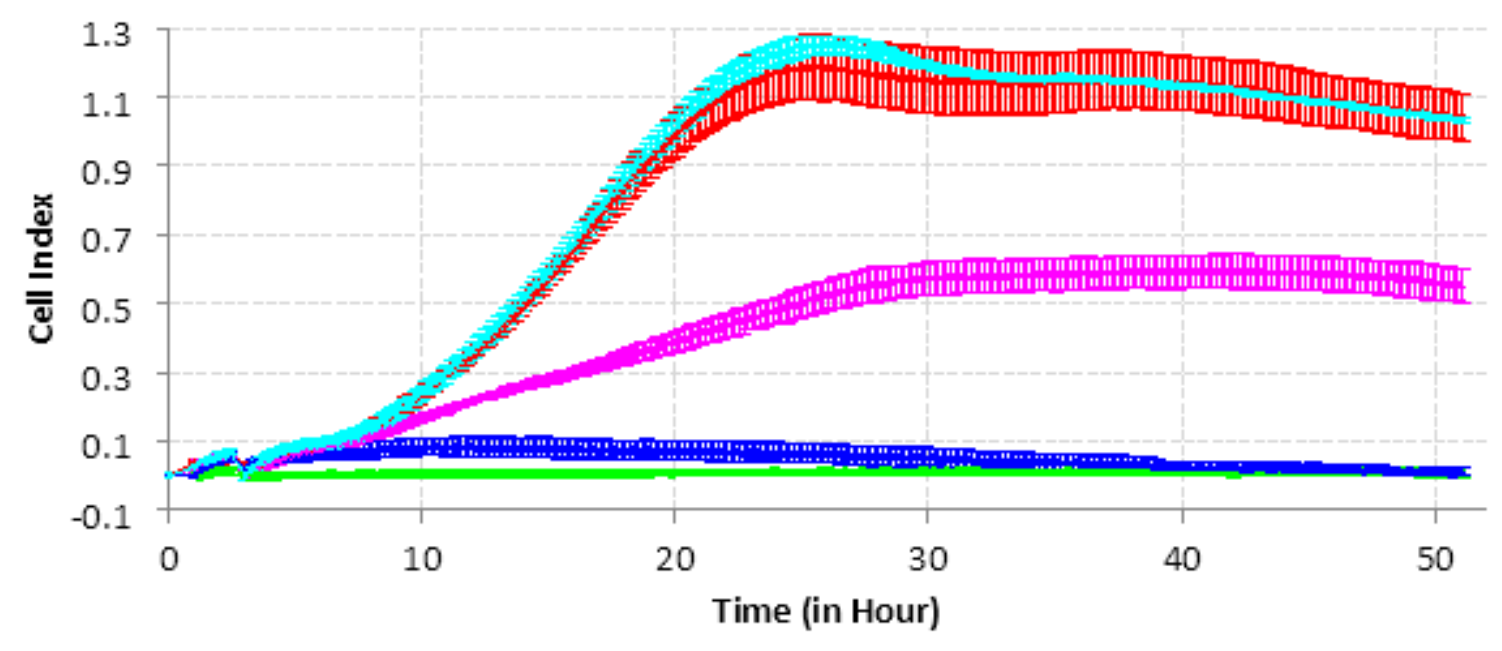

Figure 2

Time-Cl (Cell Index) plot showing the antiproliferative activity test results of Compound 9b against HeLa cell line. (The concentration unit is $\mu \mathrm{g} / \mathrm{mL}$ ).

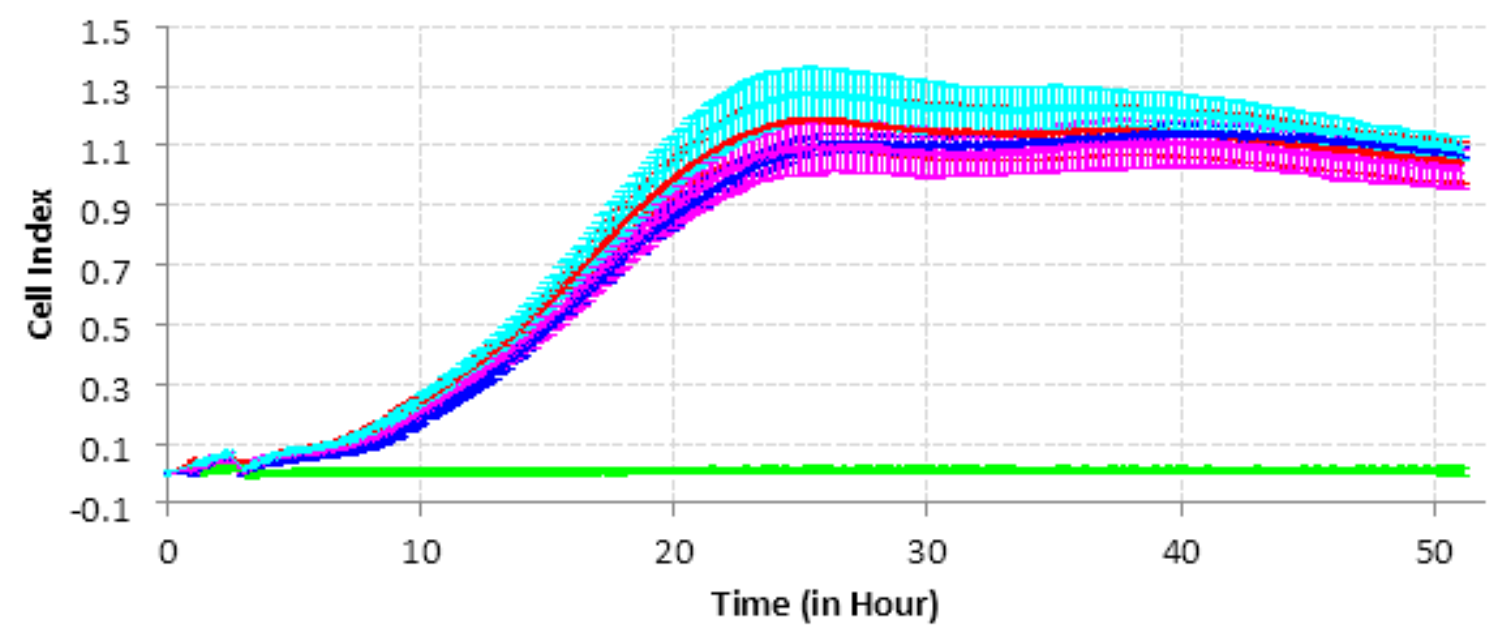

Figure 3

Adding different doses of DMSO to HeLa cell wells (The concentration unit is $\mu \mathrm{g} / \mathrm{mL}$ ). 


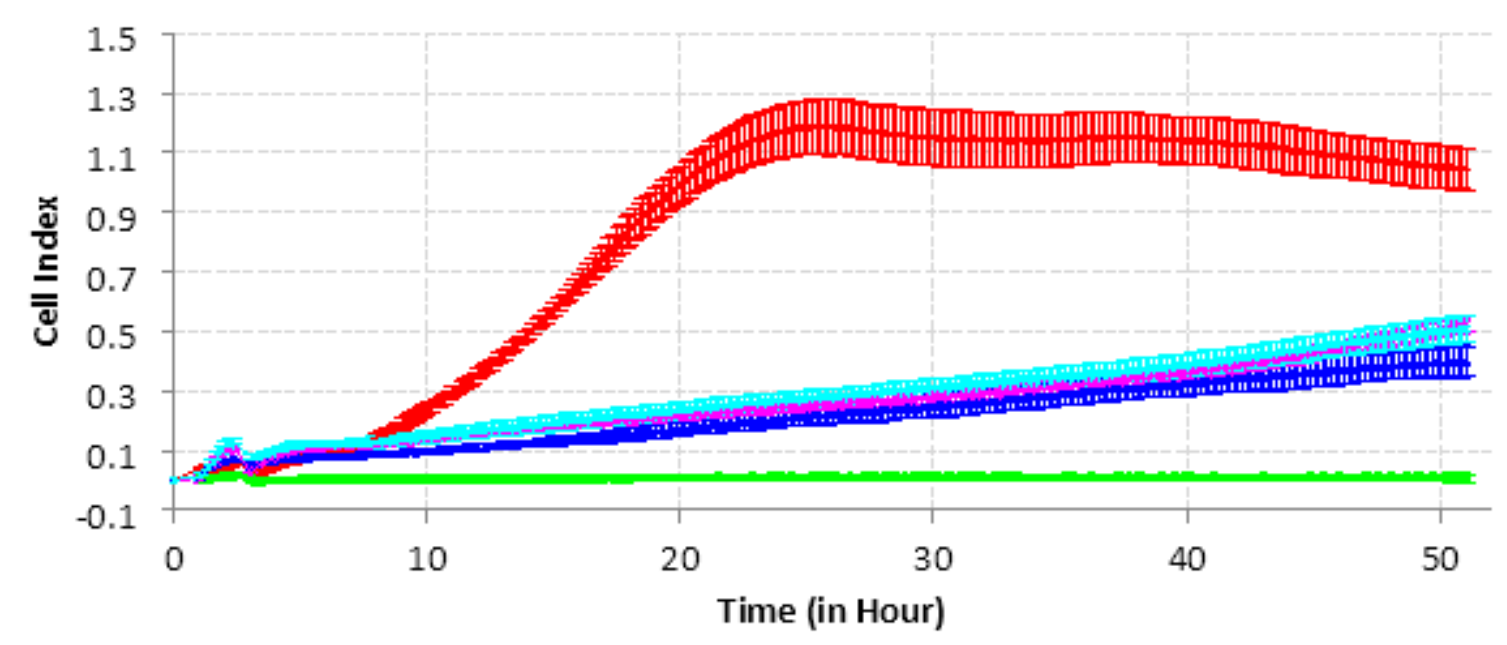

Figure 4

Effect of all 5FU doses used as positive control (The concentration unit is $\mu \mathrm{g} / \mathrm{mL}$ ).

\section{Supplementary Files}

This is a list of supplementary files associated with this preprint. Click to download.

- Scheme1.png

- Scheme2.png

- Scheme3.png

- Scheme4.png 